\title{
A lightweight vibro-acoustic metamaterial demonstrator: Numerical and experimental investigation
}

\author{
C. Claeys ${ }^{\mathrm{a}, *}$, E. Deckers ${ }^{\mathrm{a}}$, B. Pluymers ${ }^{\mathrm{a}}$, W. Desmet $^{\mathrm{a}}$ \\ ${ }^{a} K U$ Leuven, Department of Mechanical Engineering, \\ Celestijnenlaan 300 B, B-3001, Heverlee, Belgium
}

\begin{abstract}
In recent years metamaterials gained a lot of attention due to their superior noise and vibration insulation properties, be it at least in some targeted and tuneable frequency ranges, referred to as stopbands. These are frequency zones for which free wave propagation is prevented throughout the metamaterial, resulting in frequency zones of pronounced wave attenuation. Metamaterials are achieved due to addition of an, often periodic, grid of resonant structures to a host material or structure. The interaction between resonant inclusions and host structure can lead to a performance which is superior to the ones of any of the constituent materials. A key element in this concept is that waves can be affected by incorporating structural resonant elements of sub-wavelength sizes, i.e. features that are actually smaller than the wavelength of the waves to be affected. This paves the way towards compact and light vibro-acoustic solutions in the lower frequency ranges. This paper discusses the numerical design and experimental validation of acoustic insulation based on the concept of metamaterials: a hollow core periodic sandwich structure with added local resonant structures. In order to investigate the sensitivity to specific parameters in the metamaterial design and the robustness of the design, a set of variations on the nominal design are investigated. The stop bands are numerically predicted through unit cell modelling after which a full vibro-acoustic finite element model
\end{abstract}

*Corresponding author: e-mail: claus.claeys@kuleuven.be 
is applied to predict the insertion loss of the demonstrator. The results of these analyses are compared with measurements; both indicate that this metamaterials concept can be applied to combine light weight, compact volume and good acoustic behaviour.

Keywords: Metamaterials, Acoustic insulation, Insertion loss, Lightweight

\section{Introduction}

Increasing customer expectations and more restrictive legal requirements turn the acoustical behaviour of products into an important design criterion in the machine and transportation industry as well as in the construction and consumer goods sector. Ecological trends and the associated run for efficiency, however, increase the importance of lightweight design and reduce the applicability of classical (heavy) solutions to improve acoustic behaviour. In view of this challenging and often conflicting task of merging acoustical and lightweight requirements novel acoustic solutions are required. Ideally these novel solutions should be easy to design and are characterised by a low mass and compact volume along with a high reliability at an affordable cost.

Vibro-acoustic metamaterials come to the fore as possible candidates for lightweight material systems with superior noise and vibration insulation, be it at least in some targeted and tuneable frequency ranges, referred to as stop bands. Contrary to phononic crystals, stop bands in metamaterials do not rely on periodicity or Bragg scattering and work on spatial scales much smaller than the wavelength [13. The stop bands induced in metamaterials result from resonant cells arranged on a subwavelength scale and can be described based on the Fano-type interference in the structure between incoming waves and the waves re-radiated by the resonant cells [8, 11].

Previous papers of the authors study metamaterials through academic case studies; the driving parameters for vibrational stop bands are derived [5] and it is shown that vibrational stop bands can be extended to acoustic stop bands [4]. This paper focuses on translating the academic test cases to a set of practical 
demonstrators. Acoustic enclosures based on the concept of metamaterial with stop band behaviour are chosen as demonstrators. An innovative metamaterial concept is used to improve acoustic behaviour: resonant structures are added to cavities of a periodic core sandwich structure to create stop band behaviour. Different variations of the same demonstrator are designed in order to investigate robustness and sensitivity to design parameters. Numerical models are built in order to compare prediction of the stop band behaviour and insertion loss of these demonstrators with measurements.

The paper is outlined as follows. The first section introduces the concept of metamaterials through inclusion of resonant structures. The second section elaborates on the sensitivities which will be investigated through the different demonstrators. Next the potential of this metamaterial concept is assessed through unit cell modelling of the different demonstrators. The test set-up and a discussion of the modelling of this set-up and the different enclosures are the topic of the next section. The derived models are used to provide a numerical validation which can be compared to the measurement results. First the nominal acoustic enclosure is discussed; next the variations of enclosures are compared. The main conclusions are given in the last section.

\section{Metamaterial concept}

To obtain metamaterials with stop band behaviour two conditions need to be met; resonant cells have to be added to a host structure on a scale smaller than the structural wavelengths to be influenced [5] and the net sum of the forces on the hosting structure contributed by a resonator should be non-zero [17]. Metamaterials with stop band behaviour are obtained through the inclusion of resonant cells on a scale smaller than the structural wavelengths to be influenced [5]. Stop band behaviour can thus be achieved through the introduction of any system that introduces local resonant behaviour: in literature examples can be found of grids of mass-spring systems that act as resonant system [5, 12, 18. In view of engineering applications, the goal is to find resonant systems which do not conflict with other functional requirements such as structural integrity, 
low mass, use in contaminated environment, fire-resistance, ... . The kinds of resonant systems which are eligible heavily depend on the structure to which the resonant systems have to be added.

Lightweight periodic structures, such as honeycomb core sandwich panels, are becoming attractive for applications in transport and machine design due to the combination of excellent mechanical properties with a low mass. Figure 1 shows examples of sandwich structures; the core acts as spacer to create distance between the skins such that a light structure with excellent bending stiffness properties is obtained. The core has as main role to create distance between the skins as well as to resist forces perpendicular to the structure while the skin is designed to show a high in plane strength. Given the different requirements for both, often the skin is made of a different material than the core. Different core layouts are possible and two typical layouts are hexagonal and rectangular cores.

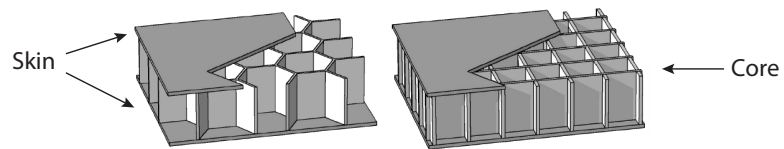

Figure 1: Examples of sandwich structures; left a hexagonal core, right a rectangular core [16].

Since these sandwich panels have internal cavities, they provide potential to include resonant systems within these cavities. This has a double advantage: (i) inclusion of resonant systems within this host structure protects the resonant systems from environmental influences and (ii) these inclusions can be added without affecting the, often excellent, stiffness properties of the structure.

A resonant system is chosen which resembles a mass-spring system; two thin legs are used to connect a heavy mass to a structure, shown in figure 21). The connection legs determine the stiffness while the thick part of the resonator determines the mass of the resonator. Figure 3 shows the addition of resonators to the cavities of a rectangular core, introducing stop band behaviour.

The design of the resonant structure as depicted in figure 2 has the following advantages: 


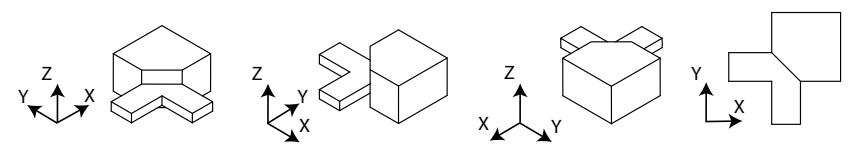

Figure 2: Resonant structure used in this paper to introduce stop band behaviour.

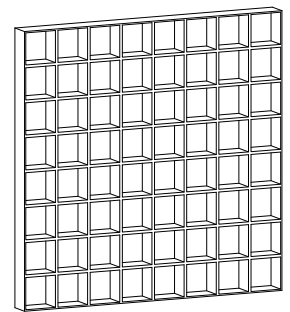

10

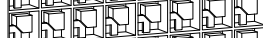

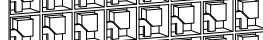

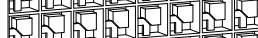

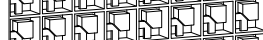

DIDT

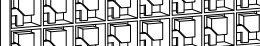

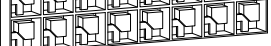

Figure 3: Rectangular core without (left) and with (right) resonant structures.

- A clear low-frequent bending mode introduces localised resonant behaviour,

- The design which keeps an analogy to spring-mass systems allows straightforward control of the resonance frequency of the first bending mode,

- The net sum of the forces to the hosting structure contributed by this first mode will be non-zero, which was stated to be a necessity for stop band behaviour [17.

Further analysis of the resonant structures and the introduction of stop band behaviour is the topic of section 5 .

A variety of different designs are possible for the host structure and resonant structures. Since this paper has set out as goal to prove the potential and feasibility of metamaterial based stop band behaviour for acoustic insulation, one possible configuration is chosen and analysed.

\section{Demonstrator design}

To prove the potential of the introduced metamaterial concept to reduce acoustic transmission, acoustic enclosures making use of resonant structures are designed. First a nominal enclosure is described. Next different variations of the same enclosures are introduced; every enclosure has as goal to either 
investigate the sensitivity to design parameters or to verify the robustness of the metamaterial concept.

\subsection{Nominal enclosure}

To obtain an unambiguous proof of concept it is crucial to have as few unknown effects in the design as possible. Connections between different components by bolts or combining parts together by gluing, is a likely source of uncertainty. In view of sensitivity studies through changes in design, it is preferred to have a demonstrator built in one part such that these unknowns are reduced.

To measure and quantify the effect of metamaterials on acoustic transmission loss different set-ups are possible. Sound transmission loss suites or dedicated cabins often require mounting of panels such that the difference in radiated acoustic power with and without mounted panel can be compared [15]. This mounting, however, introduces uncertainty in the boundary conditions.

In order to obtain a practical engineering demonstrator, it was chosen to design acoustic enclosures as open boxes which can be placed over a small loud speaker (Figure 4). The acoustic transmission loss is then determined by comparing sound radiation with and without the enclosure entirely covering a small loud speaker. This set-up has the advantage of being a light and mobile demonstrator for which the sound insulation of the enclosures can easily be compared.

The metamaterial concept, shown in Figure 3, has a rather complex geometry and topology which is hard to produce with traditional manufacturing processes. Additive manufacturing is a production process which allows producing complex parts without the need of an expensive mould, making it a suitable production process for prototype design. Within the range of additive manufacturing processes, Selective Laser Sintering (SLS) is chosen to create the demonstrator. SLS is an additive manufacturing method where small powder particles are melted together by means of a laser. The melting of adjacent material particles is called sintering and by controlling the laser, a pattern can be sintered in a layer of material powder. Unlike other additive manufactur- 
ing methods, such as fused deposition modeling and stereolithography, the part being built is surrounded by unsintered powder at all times. This unsintered powder acts as a support for the next layers and facilitates building complex parts with jumps in geometry across the height of the part. The possibility of building complex parts is the main reason why SLS is chosen as production process. In view of the design of an enclosure two important characteristics are:

- Designed structures cannot be fully closed; all cavities within a structure need to be open to remove unsintered powder.

- The cost is material driven and scales linearly with the bounding box volume of the design.

The size of the enclosure is a balance between being large enough to still be representative as acoustic enclosure and to be easy measurable but small enough such that demonstrators can be produced at an acceptable cost. With this in mind the enclosure is designed such that the inner dimensions make up a cube of $100 \times 100 \times 100 \mathrm{~mm}$.

Figure 4 shows a side and bottom view of the nominal demonstrator design. It should be noted that the corners of the demonstrator are hollow and no resonators are added: as the cavities need to be open due to the production process and fully filled corners would increase the weight of the demonstrator.
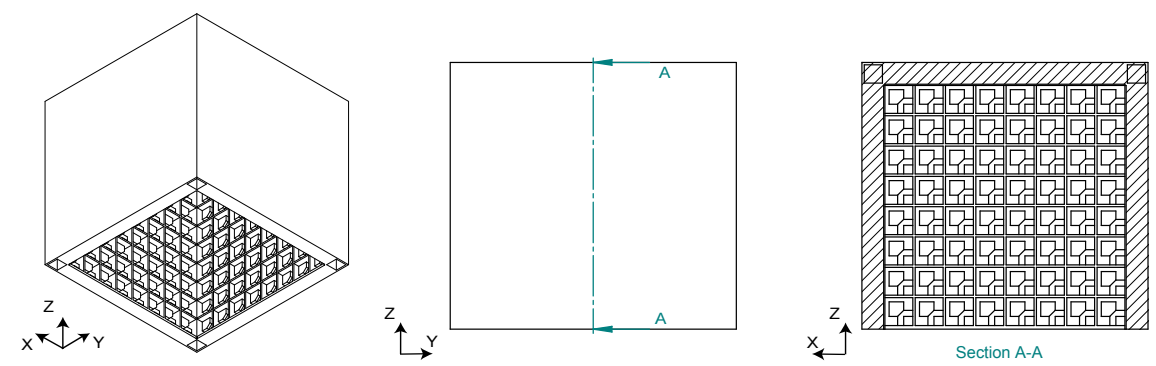

Figure 4: Nominal demonstrator design; iso (left) and side (center) and cut (right) view.

The dimensions of the resonant structure and the core of the host structure can be freely chosen. On the hand small resonant structures are preferred such 
that enough resonant structures are present on each face of the enclosure to ensure clear stop band behaviour, on the other hand the SLS production process requires minimal wall thicknesses of $0.8 \mathrm{~mm}$. Furthermore, the connection leg of the resonant structure should be thin enough to have a low resonance frequency and thick enough to be manufacturable trough SLS. The resonance frequency can be further lowered by increasing the mass of the resonant structure. Based on Finite Element (FE) model calculations a design is derived which meets the requirements, see section 4 .

Figure 5 shows the geometry of the unit cell of the nominal enclosure. One side of the enclosure contains $8 \times 8$ unit cells. The connection leg thickness $\left(\mathrm{t}_{\text {leg }}\right)$ and resonator mass thickness $\left(\mathrm{t}_{\text {mass }}\right)$ are $1 \mathrm{~mm}$ and $4 \mathrm{~mm}$ respectively for the first type of resonant structure discussed in this paper, for a second type of resonant structure these values will be $1.36 \mathrm{~mm}$ and $3.75 \mathrm{~mm}$ respectively. The resonant structure makes up $30 \%$ of the weight of the unit cell in both cases.

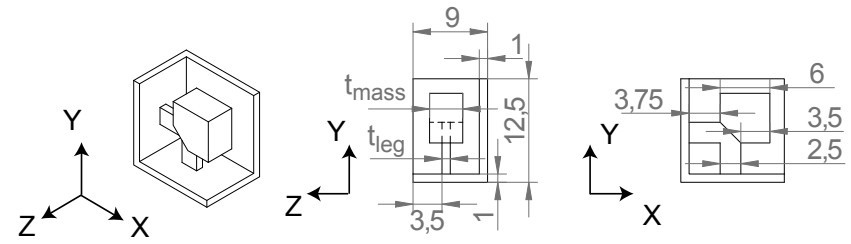

Figure 5: Dimensions $([\mathrm{mm}])$ of the unit cell of the nominal enclosure. The connection leg thickness $\left(\mathrm{t}_{\mathrm{leg}}\right)$ and resonator thickness $\left(\mathrm{t}_{\mathrm{mass}}\right)$ are dependent on the type of resonator: type $1 \mathrm{t}_{\text {leg }}=1 \mathrm{~mm}$ and $\mathrm{t}_{\text {mass }}=4 \mathrm{~mm}$, for type $2 \mathrm{t}_{\text {leg }}=1.36 \mathrm{~mm}$ and $\mathrm{t}_{\text {mass }}=3.75 \mathrm{~mm}$.

\subsection{Variations on enclosures}

In order to investigate the sensitivity to specific parameters in the metamaterial design and the robustness of the design, a set of variations on the nominal design of figure 5 are proposed.

Tables 1 and 2 give an overview of the different enclosures and their characteristic properties: thickness of the connection leg $\left(\mathrm{t}_{\mathrm{leg}}\right)$ and resonator mass $\left(t_{\text {mass }}\right)$, outer dimensions (dim.) and number of resonators (\# res). The changed parameters with respect to the nominal design are indicated with a bold font. Each design has a specific goal: 


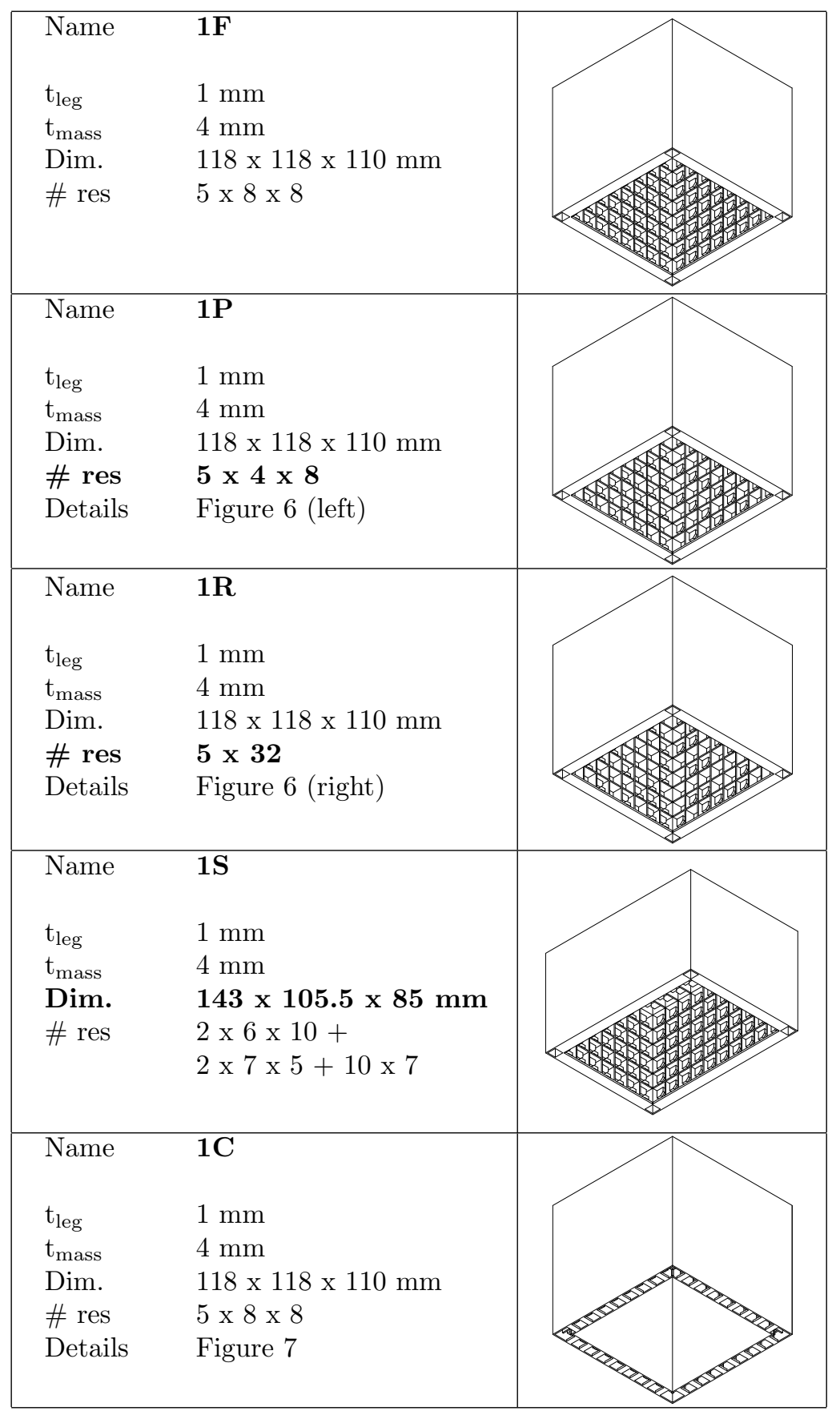

Table 1: Overview of the different enclosures, part 1. 


\begin{tabular}{|c|c|c|}
\hline $\begin{array}{l}\text { Name } \\
\mathrm{t}_{\text {leg }} \\
\mathrm{t}_{\mathrm{mass}} \\
\text { Dim. } \\
\# \text { res }\end{array}$ & $\begin{array}{l}\text { 1I } \\
1 \mathrm{~mm} \\
4 \mathrm{~mm} \\
118 \times 118 \times 110 \mathrm{~mm} \\
5 \times 8 \times 8\end{array}$ & 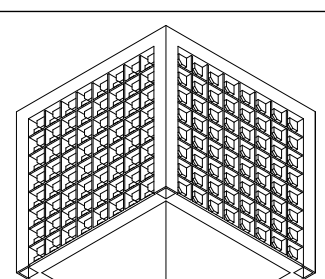 \\
\hline $\begin{array}{l}\text { Name } \\
t_{\text {leg, } 1} \\
t_{\text {mass }, 1} \\
t_{\text {leg, } 2} \\
t_{\text {mass }, 2} \\
\text { Dim. } \\
\text { \# res 1 } \\
\text { \# res 2 }\end{array}$ & $\begin{array}{l}\mathbf{2 M} \\
1 \mathrm{~mm} \\
4 \mathrm{~mm} \\
1.38 \mathrm{~mm} \\
3.75 \mathrm{~mm} \\
118 \times 118 \times 110 \mathrm{~mm} \\
\mathbf{5} \times \mathbf{4} \times \mathbf{8} \\
\mathbf{5} \times \mathbf{4} \times \mathbf{8}\end{array}$ & \\
\hline $\begin{array}{l}\text { Name } \\
\mathbf{t}_{\text {leg }} \\
\mathbf{t}_{\text {mass }} \\
\text { Dim. } \\
\# \text { res }\end{array}$ & $\begin{array}{l}\mathbf{2 F} \\
\mathbf{1 . 3 8} \mathbf{m m} \\
\mathbf{3 . 7 5} \mathbf{~ m m} \\
118 \times 118 \times 110 \mathrm{~mm} \\
5 \times 8 \times 8\end{array}$ & \\
\hline $\begin{array}{l}\text { Name } \\
\mathrm{t}_{\text {wall }} \\
\text { Dim. } \\
\text { \# res }\end{array}$ & $\begin{array}{l}\mathbf{1 N} \\
3.5 \mathrm{~mm} \\
118 \times 118 \times 110 \mathrm{~mm} \\
\text { none }\end{array}$ & \\
\hline
\end{tabular}

Table 2: Overview of the different enclosures, part 2. 


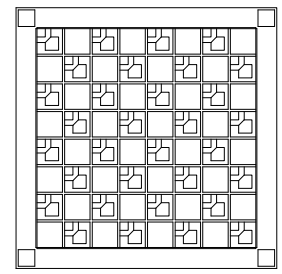

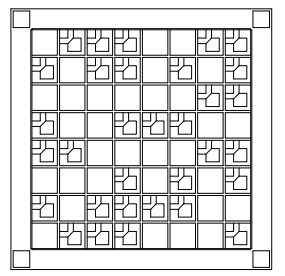

Figure 6: Bottom view of the enclosure $1 \mathrm{P}$ (left) and $1 \mathrm{R}$ (right).

1F: The nominal enclosure as described in the previous chapter.

1P: Design to investigate the importance of the number of resonators in an enclosure; half of the resonators are removed in a periodic manner. In the even rows, starting from the bottom, the resonant structures on even positions are removed, in uneven rows the uneven positioned resonant structures are removed. Figure 6 (left) shows the layout of the top plane of the enclosure as seen from the bottom.

1R: Design to assess the importance of periodicity in an acoustic metamaterial; half of the resonant structures are randomly removed in each side plane of the enclosure. The layout for the top plate, seen from the bottom, is shown on the right in figure 6 , the side planes are similar but the pattern of the removed resonators for each side is different. The acoustic characteristics of this enclosure should be compared to enclosure $1 \mathrm{P}$.

1S: Design to address the shape of the enclosure; the metamaterial concept should improve transmission loss independent of the outer dimensions of the enclosure. The dimensions of the side planes of the enclosure are changed according to table 1

1C: Design to show the potential of the metamaterial concept in case the resonant structures need to be shielded from the environment. Upon addition of a second skin the design of the unit cell needs to be adapted since the production method requires all cavities to be open. To this means, the side wall of the structure is not fully closed to achieve open cavities, as 
indicated in figure 7

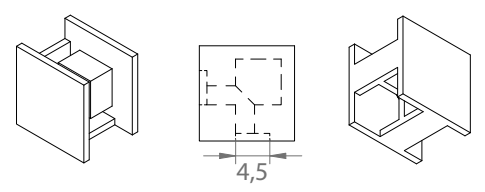

Figure 7: Unit cell of enclosure 1C.

1I: Design to discriminate the metamaterial effect from possible absorption effects due to the change in wetted interior surface. By adding a skin on the inside instead of the outside of the enclosure the inner surface of this enclosure is similar to the inner surface of the enclosure without resonators.

2M: Design to assess the effect of a combination of two different resonant structures. The second type of resonant structures is added in the voids of the $1 \mathrm{P}$ configuration, such that both structures follow each other in an alternating fashion. The resonance frequency of the second resonator is increased by increasing the connection leg thickness $\left(\left(t_{\text {leg }}\right)\right)$ and decreasing the resonator mass thickness $\left(\left(t_{\text {mass }}\right)\right)$ in the resonator geometry of figure 57. The changes in thicknesses are performed such that the total mass of enclosure $1 \mathrm{~F}$ and $2 \mathrm{M}$ are the same. The thicknesses are given in table 2 and are chosen such that the difference in thicknesses are large enough to be fabricated but small enough to have an acceptable frequency shift between both resonant systems.

2F: Design to investigate the effect of changing the resonance frequency of the resonant structure. The second type of resonant structure of enclosure $2 \mathrm{M}$ is used as resonant structure. Since both resonant structures have the same mass, the total mass of enclosure $1 \mathrm{~F}$ and $2 \mathrm{~F}$ are the same but the resulting stop band should be higher in frequency.

1N: Design to benchmark the transmission loss of the acoustic metamaterial enclosures to an enclosure with the same mass and dimensions, but with- 
out resonators. The enclosure has no resonant structures and has flat side panels; the thickness of the side panels is such that the weight is similar to the weight of enclosure $1 \mathrm{~F}$.

\section{Stop band analyses}

Stop bands can be predicted for periodic structures through unit cell modelling and the application of the Bloch-Floquet boundary conditions. This section first briefly discusses unit cell modelling, next the structural FE models of the unit cells are derived in order to compute the dispersion curves of the infinite periodic structures corresponding to the different enclosures presented in table 1 and 2 and to investigate the stop band behaviour. The acoustic effect of these structural stop bands will be discussed in next section.

\subsection{Unit cell modelling}

From literature it is known that wave propagation through infinite periodic structures can be investigated through unit cell modelling and application of the Bloch Floquet boundary conditions [3, 10. Based on an undamped FE model of the unit cell and the application of periodicity boundary conditions, dispersion surfaces for freely propagating waves in an infinite periodic structure can be derived. Frequency zones for which no solutions are found, correspond to frequency zones without free wave propagation and thus a stop band region.

Since the analysed structure is periodic, also the dispersion surface are periodic and hence not the entire wave domain must be investigated. A periodic zone in the wave domain is called a Brillouin zone, and only the first Brillouin zone needs to be examined [3, 9. Additional simplification is possible in the case of symmetry within the unit cell itself. Exploiting this symmetry, the first Brillouin zone can be further reduced to an irreducible Brillouin zone which is the smallest zone in the wave domain that contains all information [6, 14].

In literature a further reduction in computational cost is often achieved by limiting the variation of the wave vector along the contour of the irreducible Brillouin zone. It is commonly accepted in literature that the maxima and 
minima of each dispersion surface can be found on this boundary and that the resulting dispersion curves contain sufficient information to identify stop bands [14]. The cross section of the dispersion surface along this contour makes up a dispersion diagram.

When studying square unit cells which are symmetric around the diagonal axis wave propagation in $x$ - and $y$-direction behaves similarly; the irreducible Brillouin zone is thus triangular with as corner points the wave vectors which results in phase shifts $(0,0),(\pi, 0)$ and $(\pi, \pi)$ across the $(x, y)$-direction of the unit cell [6]. These corner points are systematically marked with the notation $O, A$ and $B$. When investigating the irreducible Brillouin contour, only the edges of the triangle with corner points $\mathrm{O}, \mathrm{A}, \mathrm{B}, \mathrm{O} \mapsto(0,0),(\pi, 0),(\pi, \pi),(0,0)$ need to be investigated. An example of the interpretation of such a dispersion diagram is given in the next section.

\subsection{Nominal enclosures}

Figure 8 depicts the FE model of the unit cell of the nominal enclosure. Linear Quad4 elements are used to represent the resonant structure, core and skin. The resonator mass has a larger thickness than the connection legs; different properties are assigned to both element groups. The resonator mass element group is depicted in grey on the right side of figure 8
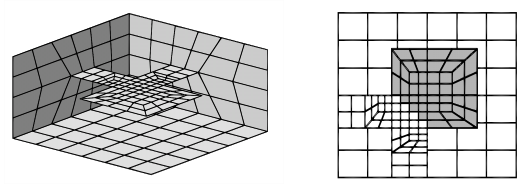

Figure 8: FE model of the unit cell of the nominal enclosure. The grey elements in the top view (right) represent the resonator mass.

The material characteristics of the enclosures are given in table 3 . Since the main goal of the simulations is to get an insight into the working principle of metamaterials based on resonant structures rather than obtaining a detailed material model, isotropy and linear behaviour of the material are assumed. 
In order to calculate the dispersion diagrams for $2 \mathrm{D}$ infinite panels, free wave propagation is assumed and damping is not included in the unit cell model.

\begin{tabular}{ccc}
\hline Name & Symbol & Value \\
\hline Young's modulus & $E$ & $1.65 \mathrm{MPa}$ \\
Density & $\rho$ & $950 \frac{\mathrm{kg}}{\mathrm{m}^{3}}$ \\
Poisson's ratio & $\nu$ & 0.4 \\
\hline
\end{tabular}

Table 3: Material characteristics of the material of the enclosures in the numerical simulations.

The resonant structure is designed to have one pronounced low-frequency bending mode followed by subsequent modes higher in frequency. Figure 9 shows the first two modes of the free unit cell which can be related to modes of the resonant structure. At the left, the bending mode is shown, which occurs at a frequency of $1272 \mathrm{~Hz}$, the next mode, shown at the right, occurs at $5052 \mathrm{~Hz}$ This large shift in frequency between the modes allows correlating stop band behaviour with a dedicated mode. Furthermore, the interation of the resonant structure with the host structure will clearly lead to a non-zero net force contribution for the first mode. Convergence of the model was validated against a refined unit cell model of 6766 nodes and 6576 elements. The used model with 198 nodes and 168 elements has an accuracy of $1 \%$ on the first mode and $1.5 \%$ on the second mode.

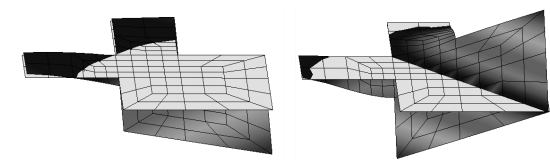

Figure 9: Undeformed mesh (light) and the deformation of the first modes (dark) of the resonant structures. Left: bending mode at $1272 \mathrm{~Hz}$. Right: torsional mode at $5052 \mathrm{~Hz}$.

\subsection{Variation of enclosures}

The FE model of the unit cell of enclosure $1 \mathrm{~F}$ allows deriving unit cell models for all other enclosures, except for the unit cells of enclosure $1 \mathrm{C}$ and $1 \mathrm{~N}$. The following description lists the different unit cells for every enclosure: 
1F-1S-1I: For the calculation of the dispersion curves the equivalent infinite flat counterpart of the enclosures is studied. The equivalent infinite counterparts of enclosure $1 \mathrm{~F}, 1 \mathrm{~S}$ and $1 \mathrm{I}$ are the same, meaning that the unit cell of figure 8 can be used for the stop band analysis of these enclosures.

1P-1R: The unit cell of enclosure 1P consists of two cells of the host structure where in only one of these cells a resonant structure is added. Enclosure $1 \mathrm{R}$ has no unit cell due to the random manner in which the resonant structures are removed; an average unit cell, however, would be similar to the unit cell of enclosure 1P. Figure 10 shows this unit cell. In this case the directions of periodicity, $\boldsymbol{d}_{1}$ and $\boldsymbol{d}_{2}$ wil not be in the $x$ - and $y$-direction anymore, but will by rotated 45 degrees around the $\mathbf{z}$-axis, as indicated in figure 11. It should be noted that this unit cell is not square anymore, the direction vectors of periodicity, however, are still orthogonal and have an equal length, meaning that direction vectors are similar as for square unit cells; the same irreducible Brillouin zone can be used but the corner points of the irreducible Brillouin contour correspond to different directions. Also the unit cell is not symmetric anymore, however, by comparing against the full dispersion surfaces, it was validated that the irreducible Brillouin contour can be used to predict the stop band frequencies correctly.

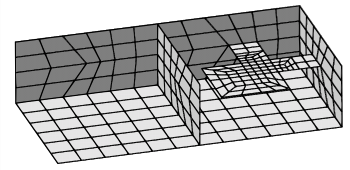

Figure 10: FE model of the unit cell of enclosure 1R and 1P.

1C: Enclosure 1C requires a different mesh of the core and skins; the corresponding FE model is shown in figure 12. Due to the changed thicknesses, the first modes of the unit cell were shown to have an accuracy of $1.5 \%$ with respect to refined models.

2M: Enclosure 2M is made up of a repetition of 2 unit cells of enclosure $1 \mathrm{~F}$ in 

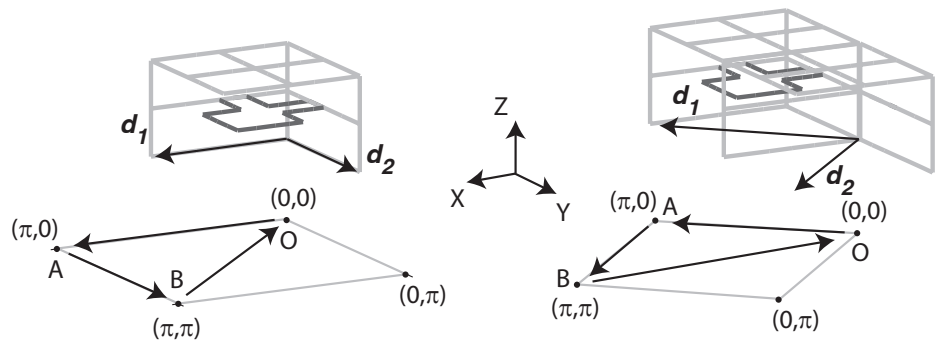

Figure 11: Interpretation of the irreducible Brillouin contour $O A B O$ for the square unit cells and the unit cell of enclosure $1 \mathrm{R}, 1 \mathrm{P}$ and $2 \mathrm{M}$. The direction vectors of periodicity $\boldsymbol{d}_{1}$ and $\boldsymbol{d}_{2}$ for both unit cell types are indicated with labelled black arrows.

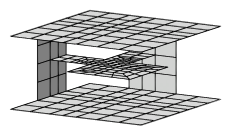

Figure 12: FE model of the unit cell of enclosure 1C.

which one of both resonant structures is replaced with a resonant system with a thicker connection leg and thinner resonator mass. These changes in thicknesses correspond to changes in the properties of the corresponding elements and thus no new mesh is required (Fig 13 ).

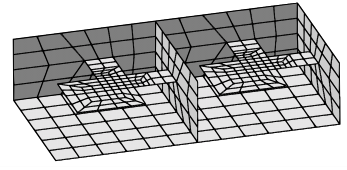

Figure 13: FE model of the unit cell of enclosure 2M.

2F: In case of enclosure $2 \mathrm{~F}$ only the second resonant structure is present. The FE model of enclosure $1 \mathrm{~F}$ is used and the thickness of the connection legs and the resonator mass are changed accordingly. The first bending mode of the resonant systems rises to a frequency of $1746 \mathrm{~Hz}$ while the second mode rises to $7388 \mathrm{~Hz}$.

$1 \mathrm{~N}$ : The unit cell of enclosure $1 \mathrm{~N}$ corresponds to the unit cell of a flat plate, which is already thoroughly investigated in literature [5]. It was shown that bare plates do not show stop band behaviour in the low-frequency 
regime.

\section{Unit cell analyses}

Once the different models are built, the dispersion curves for all enclosure types can be derived. This section starts with the description of the dispersion curves of an infinite host structure without resonant structures. After gaining insight in the host structure, the effect of adding resonant structures is discussed.

\subsection{Host structure}

Figure 14 shows the dispersion curves of the host structure of enclosure $1 \mathrm{~F}$ without resonant structures. The right side of figure 14 shows a representation of the unit cell; only some characteristic lines of the unit cell are displayed. This simplified representation of the unit cell is chosen in order not to overload the figures when visualising multiple unit cells together as in the bottom of the figure.

On the bottom of figure 14 the displacements of $10 \times 2$ unit cells are shown corresponding to the markers on the dispersion curves of the periodic structure. The markers are positioned on a point of the Brillouin contour in section $O A$, and thus waves propagating in the $x$-direction are found. Inspection of the displacements shows three different wave types propagating through the structure: a bending wave $(\nabla)$, an in-plane shear wave $(\diamond)$ and a longitudinal dilatational wave $(\square)$. It is clear that the three dispersion curves that start in the origin correspond to these three different wave types. It can be noted that since the visualisation of the displacement of the dilatational waves is already at higher frequencies, the dynamic behaviour of the core walls starts showing; the small half sines in $y$-direction on the side of each unit cell correspond to the visualisation of the core displacement.

In view of acoustics, in-plane waves can be important as transmission method for vibration energy, however, these waves are not efficient as acoustic radiators since the surrounding air is not influenced by their motion; only the dispersion 

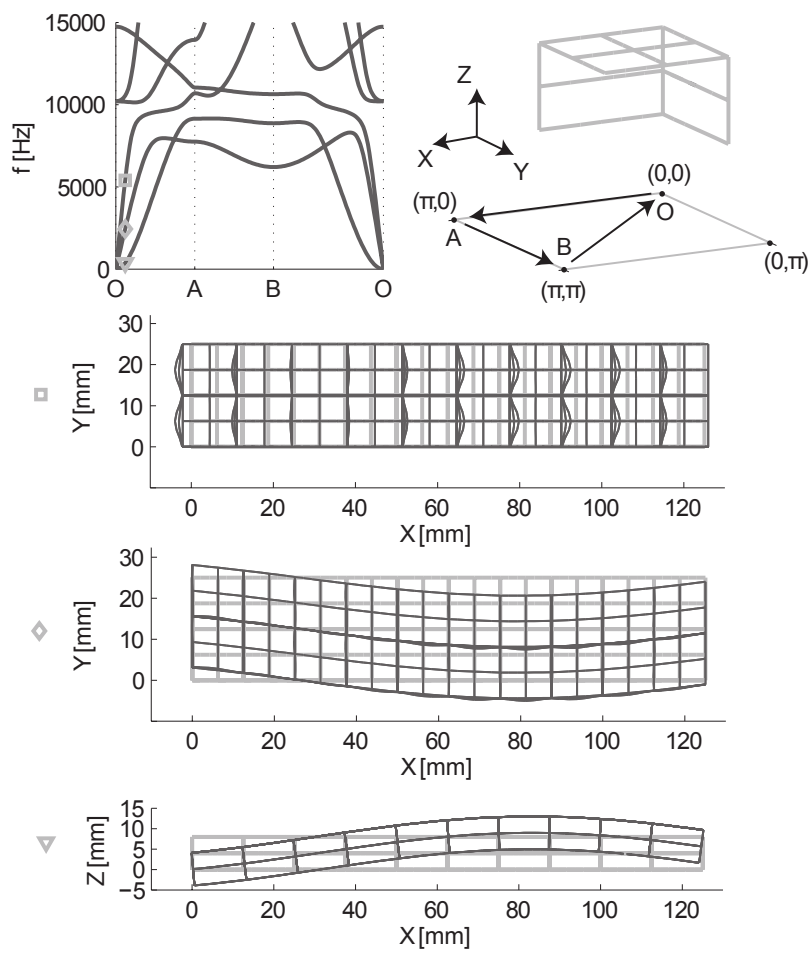

Figure 14: Dispersion curves (left) for the unit cell of enclosure 1F without resonant structure (right top) according to the Brillouin contour depicted below. Bottom: Displacement of the infinite periodic structure corresponding to the markers on the dispersion curves in the top figure. A section, with the size of 10 unit cells in $\mathrm{x}$-direction and 2 unit cells in y-direction, of the infinite periodic structure is visualised. The undeformed structure $(-)$ is overlayed by the deformed structure (-). From top to bottom an in-plane longitudinal dilatational (top view), in-plane shear wave (top view) and bending wave (side view) can be seen. 
curves corresponding to bending waves should be considered for coincidence investigations. Since the expected stop band of the metamaterial design is around $1200 \mathrm{~Hz}$ and since in-plane motion does not directly contribute to acoustic radiation, only the dispersion curve related to bending motion in the frequency zone 0 to $2500 \mathrm{~Hz}$ is shown on the following dispersion diagrams.

\subsection{Nominal enclosure}

Figure 15 shows the bending wave dispersion curves for the unit cell of the nominal enclosure $1 \mathrm{~F}$ together with the dispersion curves of the host structure without resonant structures of figure 14. The dispersion curves are similar except in the region of the resonance frequency of the resonant system; a stop band opens up from $1065 \mathrm{~Hz}$ up to $1226 \mathrm{~Hz}$.

Figure 16 repeats the dispersion curves of the nominal enclosure and visualises the motion of the structure at some points of the Brillouin contour, indicated by markers, to give insight in the nature of the stop band. The motion of the structure is represented by the displacement of $3 \times 2$ unit cells. To improve interpretation of the displacements, a thin black line indicates the position of the unperturbed system.

The dispersion curve of bending motion splits up into two; a curve corresponding to in-phase motion of the resonators and the host structure $(\nabla)$ and a curve corresponding to out-of-phase motion of both ( $\square$ ). On the flat part of the dispersion branch the periodic structure shows very little deformation and all movement is localised in the resonators $(\diamond, \circ)$. These results correspond to the conclusions of previous research [4] where it was stated that inclusion of resonant systems leads to a mode split: one set of in-phase modes and one set of out-of-phase modes.

\subsection{Variation of enclosures}

Table 4 gives an overview of the dispersion curves of the different enclosures. For each realisation, the relative mass addition of the resonant structure to the total weight of the unit cell is listed (Rel. mass) together with the limiting frequencies of the stop band and the relative stop band width with respect to 


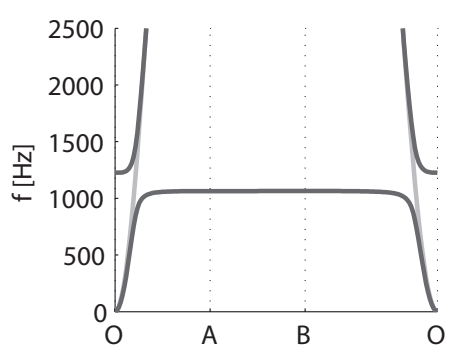

Figure 15: Dispersion curves of the nominal enclosure $(-)$ in comparison with the dispersion curves of the host structure $(-)$.
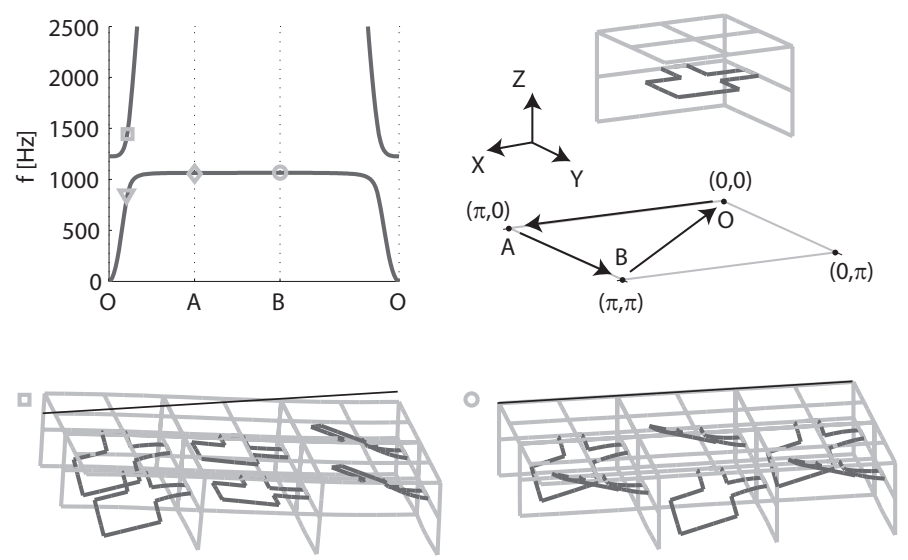

$\nabla$
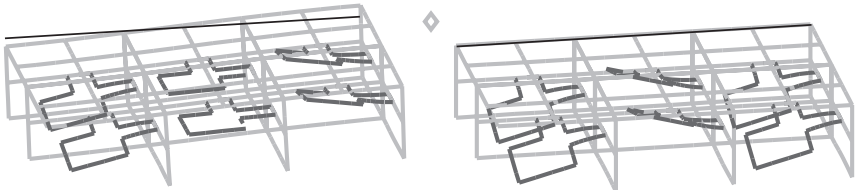

Figure 16: Dispersion curves (left) for the unit cell of the nominal enclosure $1 \mathrm{~F}$ according to the Brillouin contour depicted on the right. Bottom: Displacement patterns of $3 \times 2$ unit cells corresponding to the markers on the dispersion curves. The position of the undeformed structure is indicated with a thin black line $(-)$. 
the centre frequency of the stop band $\left(\frac{\Delta f_{\text {stop band }}}{f_{\text {centre }}}\right)$. A detailed study of these results shows:

1F-1I-1S: Enclosures 1F, 1S and 1I are constituted of the same unit cell and are thus characterised by the same dispersion curves. For these realisations, a $30 \%$ mass addition leads to a relative stop band width of $14 \%$.

1P-1R: Due to the removal of half the resonant structures, the relative mass addition of the resonant structure drops with $58 \%$, the relative stop band width drops with almost an equal amount of $57 \%$. As discussed previously, the corner points of the irreducible Brillouin contour correspond to different directions for this unit cell, as detailed in figure 11 .

1C: The stop band width of enclosure $1 \mathrm{C}$ is only $75 \%$ of the stop band width of enclosure $1 \mathrm{~F}$. This effect is partly explained by the difference in relative mass of the resonant structure to the host structure; the relative mass of the resonant structure lowers from $30 \%$ for configuration $1 \mathrm{~F}$ to $28 \%$ for enclosure $1 \mathrm{C}$ due to the difference in hosting structure. A more crucial effect on the stop band width seems to be the difference in stiffness of the hosting structure; the core is less stiff due to the open section in the core walls, which influences both the stiffness of the overall structure as the stiffness of the connection between the resonant structure and host structure. As can be seen in figure 17 the dispersion curves of enclosure $1 \mathrm{C}$ is less steep and the second dispersion curve starts out less flat as the second dispersion curve of enclosure $1 \mathrm{~F}$.

2M: Enclosure 2M is made up of a repetition of two different resonant structures which both have the same weight. Both resonant systems lead to a stop band in the region of their resonance frequencies. Although both resonant structures have the same weight, the relative width of the stop band corresponding to the resonant structure with the highest resonance frequency is larger. This might be explained by a difference in dynamic mass; during the first stop band, the second resonant system moves in- 


\begin{tabular}{|c|c|c|c|c|c|}
\hline $\begin{array}{l}\text { Enclosures } \\
\text { Rel. mass } \\
\text { Stop band } \\
\frac{\Delta \mathrm{f}_{\text {stop band }}}{\mathrm{f}_{\text {centre }}}\end{array}$ & $\begin{array}{l}1 \mathrm{~F}, 1 \mathrm{I}, 1 \mathrm{~S} \\
30 \% \\
1065-1226 \mathrm{~Hz} \\
14 \%\end{array}$ & 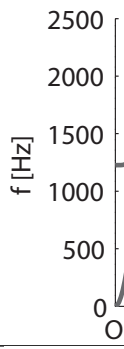 & $\mathrm{A}$ & B & 0 \\
\hline $\begin{array}{l}\text { Enclosures } \\
\text { Rel. mass } \\
\text { Stop band } \\
\frac{\Delta \mathrm{f}_{\text {stop band }}}{\mathrm{f}_{\text {centre }}}\end{array}$ & $\begin{array}{l}1 \mathrm{P}, 1 \mathrm{R} \\
17.5 \% \\
1064-1151 \mathrm{~Hz} \\
7.9 \%\end{array}$ & 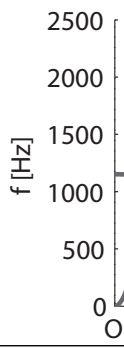 & A & B & $\mathrm{O}$ \\
\hline $\begin{array}{l}\text { Enclosure } \\
\text { Rel. mass } \\
\text { Stop band } \\
\frac{\Delta \mathrm{f}_{\text {stop band }}}{\mathrm{f}_{\text {centre }}}\end{array}$ & $\begin{array}{l}1 \mathrm{C} \\
28 \% \\
1061-1173 \mathrm{~Hz} \\
10 \%\end{array}$ & 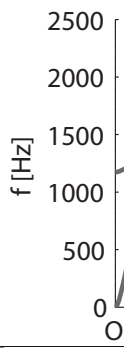 & A & B & $\mathrm{O}$ \\
\hline $\begin{array}{l}\text { Enclosure } \\
\text { Rel. mass } \\
\text { Stop band } \\
\frac{\Delta \mathrm{f}_{\text {stop band }}}{\mathrm{f}_{\text {centre }}}\end{array}$ & $\begin{array}{l}2 \mathrm{M} \\
15 \% \\
15 \% \\
1064-1128 \mathrm{~Hz} \\
1566-1699 \mathrm{~Hz} \\
5.8 \% \\
8.2 \%\end{array}$ & 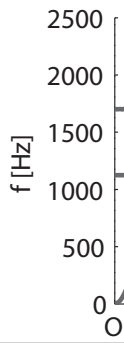 & A & B & $\frac{1}{0}$ \\
\hline $\begin{array}{l}\text { Enclosure } \\
\text { Rel. mass } \\
\text { Stop band } \\
\frac{\Delta \mathrm{f}_{\text {stop band }}}{\mathrm{f}_{\text {centre }}}\end{array}$ & $\begin{array}{l}2 \mathrm{~F} \\
30 \% \\
1569-1799 \mathrm{~Hz} \\
13.7 \%\end{array}$ & 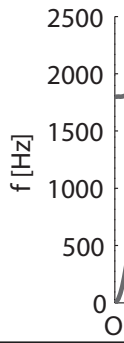 & A & B & 0 \\
\hline
\end{tabular}

Table 4: Overview of the stop band behaviour of the different enclosures. 


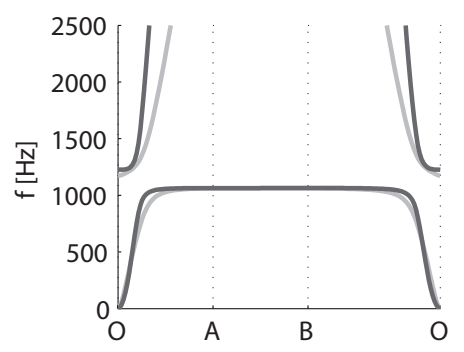

Figure 17: Dispersion curves of enclosure 1F (-) in comparison with the dispersion curves of enclosure $1 \mathrm{C}(-)$.

phase with the host structure leading to a larger dynamic mass of the combination of host structure and second resonant system. During the stop band of the second resonant system however, the first resonant system moves in out-of-phase with the host structure, making the host structure combined with the first resonant structure dynamically lighter. The apparent mass against which the first resonant structure has to act is thus larger than for the second resonant structure.

$\mathbf{2 F}$ : The dispersion curves of enclosure $2 \mathrm{~F}$ indicates a relative stop band width of $13.7 \%$ for a mass addition of $30 \%$. This result is similar to the results for enclosures $1 \mathrm{~F}, 1 \mathrm{~S}$ and $1 \mathrm{I}$.

The following trends can be derived based on these dispersion diagrams:

- In all enclosures stop bands are found due to the addition of a resonant structure.

- The stop band is for each unit cell in the frequency region of the resonance frequency of the resonant structure.

- The lower frequency of stop bands caused by the same types of resonant structure are almost equal, changes in unit cell layout seem mainly to affect the upper frequency of the stop band. 


\section{Numerical model for insertion loss prediction}

The previous section indicates stop band behaviour in the enclosures. In this section full FE models of the enclosures are derived in order to numerically predict the resulting acoustic behaviour of these enclosures. First a description of the test set-up and a discussion of the modelling of this set-up is given. Next the models of the different enclosures are explained.

\subsection{Model of the test set-up}

In view of an engineering demonstrator, acoustic enclosures were chosen as proto-types. These enclosures can be placed over a small loudspeaker such that the acoustic characteristics of each enclosure can be measured.

The test set-up consists of a small loudspeaker placed on a wooden plate, shown in figure 18 . The wooden plate is placed on an iron support in the centre of a semi-anechoic chamber. Between the wooden plate and the loudspeaker, a small $1 \mathrm{~mm}$ trim-like piece of fabric is placed; the trim covers the cable of the loudspeaker which runs in a small split in the plate.
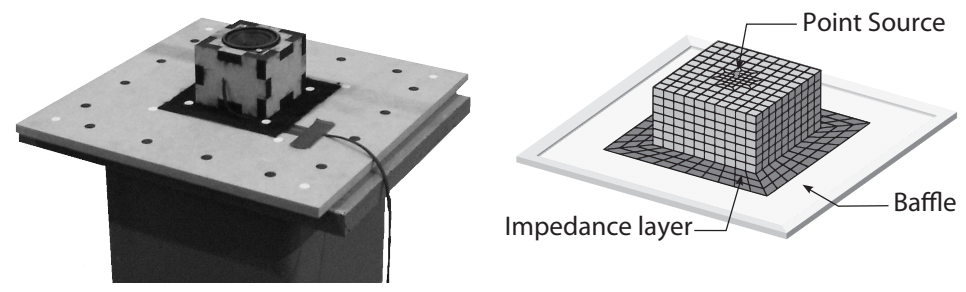

Figure 18: Picture of the test set up (left) and the model of this set up (right).

The wooden plate is modelled as a baffle: an infinite plane which is acoustically perfectly reflecting. The model is shown in figure 18. This approach is justified since the acoustic power is measured above the wooden plate and the set-up is positioned within a semi-anechoic chamber. An impedance layer in the plane of the baffle reflects the piece of trim on the wooden plate; a real impedance of $1.57810^{4} \frac{\mathrm{kg}}{\mathrm{m}^{2} \mathrm{~s}}$ corresponding to a sound absorbing coefficient of 0.1 [15] is added. The piece of trim has a size similar to the size of the acoustic enclosure, for enclosure $1 \mathrm{~S}$ a larger piece of trim of the same material is 
used. The impedance layer in the numerical model covers the part of the baffle between the model of the loudspeaker and the enclosure.

The loudspeaker is modelled as a rigid perfectly reflecting box with the same dimension as the loudspeaker: $73 \times 73 \times 55 \mathrm{~mm}$. The speaker mesh and the impedance boundary have a maximal element length of $10 \mathrm{~mm}$. Following the 6 elements per wavelength rule, this mesh is acoustically valid up to above $5000 \mathrm{~Hz}$.

To model the sound source a point source, emiting a constant intensity across the frequency spectrum, is placed $5 \mathrm{~mm}$ above the centre of the upper plane of the speaker. The upper surface of the speaker mesh is refined since the point source is placed close to this surface. The radiated power of this source point will not perfectly match the radiated power by the source, but as explained in section 7 , insertion loss is used as acoustic characteristic for the enclosures, which should be indepedent of source spectrum.

The sound power is evaluated on the surface of a surrounding box of $250 \times 250 \times 190 \mathrm{~mm}$ centred around the loudspeaker; the black dots on the wooden plate, see figure 18 left, indicate the width and length of the surrounding box. The acoustic power is evaluated based on five intensity measurements, one on each side of the box, with a scanning intensity probe: a Brüel \& Kjær 1/2-inch sound intensity microphone pair type 4197 together with a $12 \mathrm{~mm}$ spacer is used as intensity probe, shown in figure 19 . This procedure was validated by comparison with the acoustic power measured based on 64 stationary intensity measurements on the box surface; the results, shown in figure 20, agree well enough in view of the trend analysis of this paper.

The numerically predicted radiated acoustic power is calculated as the sum of the radiated acoustic intensity on a hemisphere with a $0.3 \mathrm{~m}$ radius which is centred over the loudspeaker. Figure 21 depicts the mesh, consisting of 105 elements and 113 nodes with a largest element edge equal to $0.1 \mathrm{~m}$. This mesh was validated by comparison with refined meshes for the evaluation of the acoustic radiated power. 


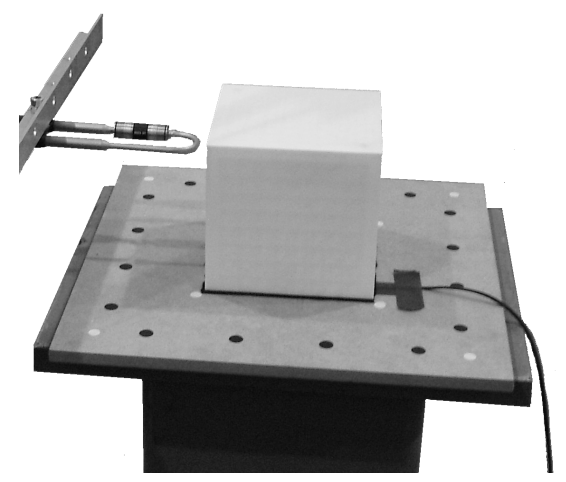

Figure 19: Picture of the test set-up during measurements on enclosure $1 \mathrm{~F}$ with the intensity in a fixed position.

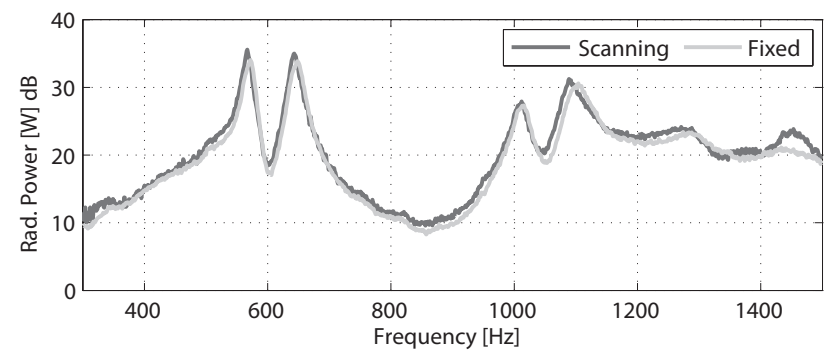

Figure 20: Measured radiated power for enclosure $1 \mathrm{~F}$ obtained with a scanning intensity probe compared to measurements with an intensity probe on a grid of fixed positions.

\subsection{Models of the enclosures}

To compute the acoustic radiated power through the enclosure under excitation of the point source within the enclosure a simulation is required in which an acoustic model of the test set-up is coupled to a vibro-acoustic model of the enclosure. To reduce computational cost, a modal-based vibro-acoustic coupling is applied; the structural modes of the enclosure are derived for a detailed structural FE mesh after which these structural modes are projected on an acoustic Boundary Element (BE) mesh of the enclosure such that a coupled vibro-acoustic simulation can be performed. These calculations are performed in the LMS VL.Acoustics software environment coupled to a Nastran structural solver and a Sysnoise acoustical solver. 


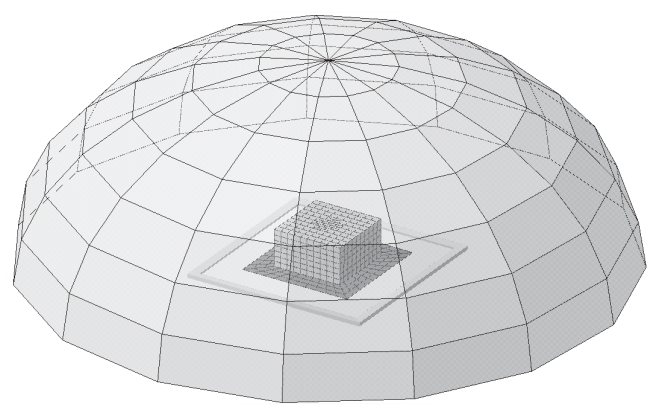

Figure 21: Mesh on a hemisphere with a $0.3 \mathrm{~m}$ radius around the model of the loudspeaker to evaluate the radiated acoustic power in the numerical model.

\subsubsection{Structural mesh}

The FE model of the structure is derived through a repetition of the unit cell described in section 5. Figure 22 shows the FE model of the nominal acoustic enclosure without outer skin. The total structural model with outer skin contains 63353 nodes and 60720 linear Quad4 elements.

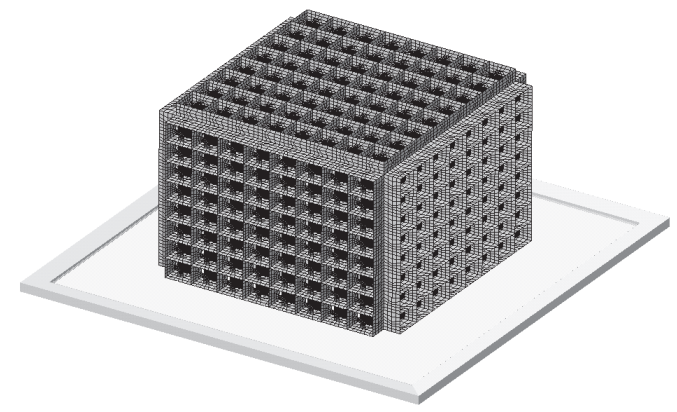

Figure 22: The structural model of the nominal enclosure without outer skin.

The element size of the structural mesh of the enclosure is driven by the smallest geometry of the unit cell. This geometry is small compared to the wavelength in the structure since the inclusion for metamaterial based stop bands needs to be smaller than the governing wavelength in the structure.

The enclosure is supported by the wooden plate and the piece of trim. This is modelled by removing the translational degrees of freedom of all elements in the bottom plane of the enclosure. The motion parallel to the wooden plate is 
influenced by the boundary as well; this is neglected in the numerical model.

To include the effect of damping both in the material as through the boundary, $1 \%$ of modal damping is applied to all the modes.

\subsubsection{Acoustic mesh}

The minimal size of the acoustic mesh is driven by the wavelength of sound in air. Since the simulations are below coincidence, the element size requirements on the acoustic mesh are less stringent than for the structural mesh. The acoustic mesh is built as a remeshing of the skin of the enclosure with $15 \times 15$ elements on each side such that the obtained element length is similar to the model of the loudspeaker. This remeshing reduces the number of elements for the outer skin from 4140 for the structural model to 1125 elements for the acoustic model. Figure 23 compares the obtained acoustic mesh (right) with the structural mesh (left).
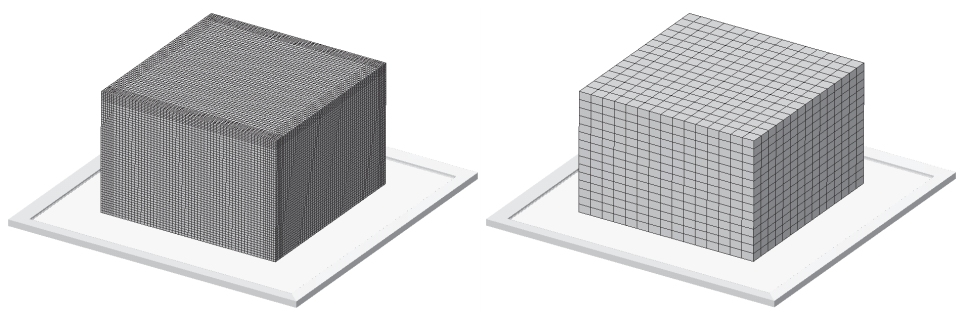

Figure 23: The structural model of the nominal enclosure with skin (left) and the acoustic model (right).

For the case of enclosure $1 \mathrm{~S}$ an acoustic mesh is derived by remeshing the outer skin of enclosure $1 \mathrm{~S}$ with a similar element size as the acoustic mesh of the nominal enclosure, shown in figure 23 (right). For the cases of enclosure 1I and $1 \mathrm{C}$ the acoustic meshes are derived by remeshing the inner skin of enclosure 1I.

To compute the acoustic radiated power through the enclosure a BE modalbased vibro-acoustic coupling is applied. This coupling requires the mode shapes, computed for the structural model, shown in figure 22 and on the left of figure 23, to be mapped onto the acoustic mesh. The equivalent displacement on a node of the acoustic mesh is calculated based on the movement of the 20 closest nodes on the skin of the structural mesh which are within a $5.5 \mathrm{~mm}$ 
radius of the node on the acoustic mesh. The contributions of these nodes of the structural mesh to the node on the acoustic mesh are weighted based on the distance between the nodes on structural and acoustic mesh.

The mapping of the modes of a structural model with skin, core and resonant structures on an acoustic mesh without core and resonant structures means that the resonant structures cannot be influenced by the acoustic medium in the simulation and the other way around, that the movement of the resonant structures will not influence the acoustic medium. This simplification was validated by comparing the obtained results with this strategy to a simulation using the full structural mesh (skin, core and resonant structure) as acoustic mesh: every node of the structural model coincided with a node on the acoustic model. The difference showed to be negligible, indicating that the mesh mapping is allowed and that the acoustic mesh size is fine enough.

This validation of the mesh mapping indicates that the surrounding air does not influence the motion of the resonators in the numerical model. In reality however, the relative motion of the resonant structure with respect to the host structure could lead to added damping by air being squeezed through the gap between resonant structure and core. The acoustic model applied does not account for possible viscosity effects.

\section{Simulation and measurement results}

Next to the numerical counterparts of the enclosures, also the enclosures themselves have to be made. As indicated in section 3 , selective laser sintering was chosen as production process for this. Fig. 24 shows the nominal enclosure as it is produced. This section analyses the predicted acoustic response of the different enclosures and compares these predictions with measurements on the fabricated enclosures.

As metric the insertion loss (IL) is considered. The insertion loss is obtained as the ratio of radiated acoustic power by the loudspeaker before an enclosure is placed over the loud speaker $\left(P_{\text {without }}\right)$ with respect to the radiated acoustic 

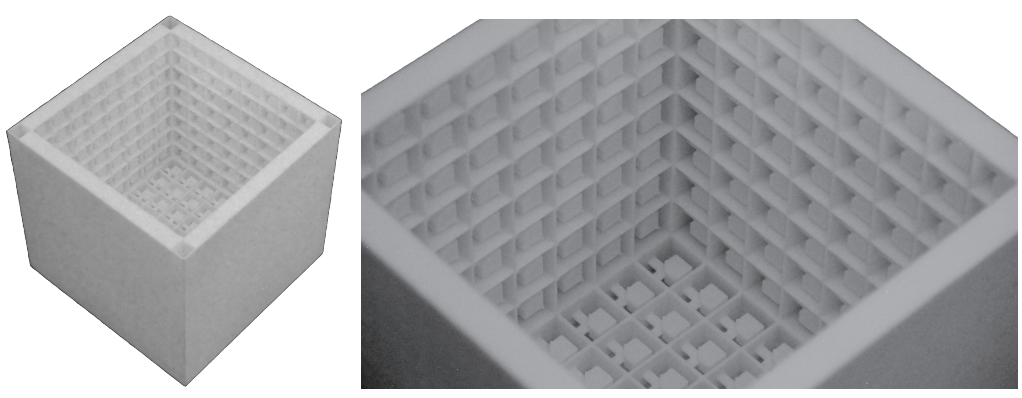

Figure 24: The nominal enclosure.

power when the enclosure is in place $\left(P_{\text {with enclosure }}\right)$ :

$$
\text { Insertion Loss }=10 \log _{10}\left(\frac{P_{\text {without }}}{P_{\text {with enclosure }}}\right) \text {. }
$$

For linear systems this metric has as advantage that the results are independent of source spectrum.

\subsection{Nominal enclosure}

Figure 25 (top) compares the insertion loss of the nominal acoustic enclosure to the IL of the host structure without resonant inclusions. The predicted stop band based on unit cell analysis is depicted with 2 narrow vertical lines. Inspection of the curves learns that:

- Both curves show a similar insertion loss at low frequencies, indicating a similar stiffness.

- The first 2 modes of the enclosure, indicated by dips in the IL, are pushed down to lower frequencies for the enclosure with resonant structures. This is both due to the stop band which pulls down the first dispersion curve, depicted in figure 15, as due to the mass addition of the resonant structures. The resonant structures add mass and have little influence on the overall stiffness of the structure; the first eigenmodes of the structure decrease in frequency.

- A strong increase in insertion loss is visible in the zone predicted by the stop band. The stop band manifests itself in a similar fashion as in the case 
of an academic example through addition of tuned vibration absorbers [4]; the maximal insertion loss is achieved in the beginning of the stop band while after the stop band a reduction in insertion loss is visible. This indicates that the principle of stop band, the inclusion of cells with resonant behaviour, mainly depends on the existence of localised behaviour and is rather insensitive to the detailed nature of this behaviour.

- For frequencies higher than $1600 \mathrm{~Hz}$, a second zone of increased insertion loss seems to manifest itself. This peak in insertion loss, however, is due to the modal behaviour of the cubic enclosure. For enclosure $1 \mathrm{~S}$ the modal density is more even throughout the frequency regime and it will be shown that this zone disappears. Also for enclosures with a different overall stiffness, such as enclosures $1 \mathrm{C}$ and $1 \mathrm{I}$, this zone will not be present since the different stiffness will lead to different modal behaviour.
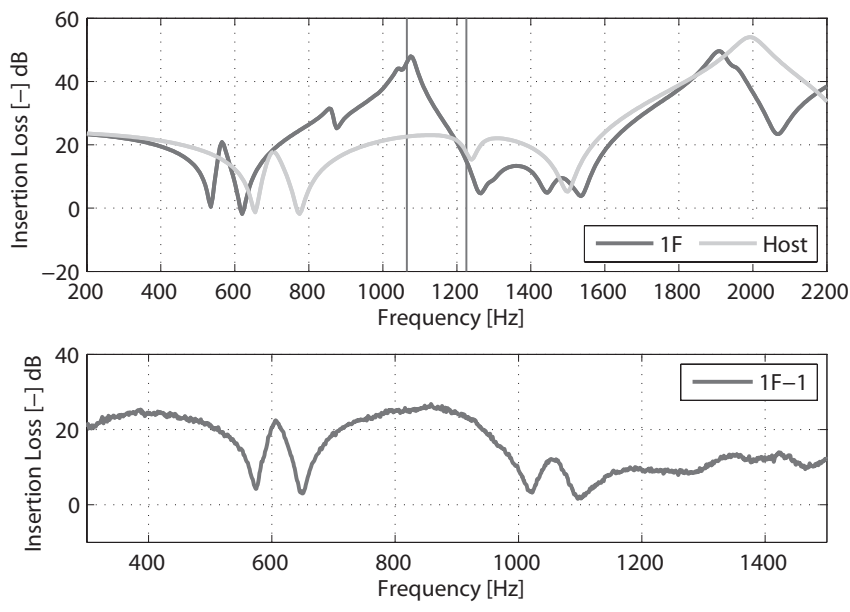

Figure 25: Top: Comparison between the numerically predicted insertion loss for enclosure $1 \mathrm{~F}$ and the host structure without resonant structure. The narrow vertical lines indicate the predicted stop band zone. Bottom: The measured insertion loss for enclosure $1 \mathrm{~F}$. The host structure without resonant structure was not produced and hence not measured.

Figure 25 (bottom) shows the measured insertion loss for enclosure 1F. The insertion loss of the enclosure build from a host structure without resonant structures was not measured since this enclosure was not produced. A zone of 
increased insertion loss is visible between 690 and $980 \mathrm{~Hz}$. Comparison with the predicted numerical results shows a relative poor correspondence of the predicted stop band effect. Although a high insertion loss is achieved, still a relative large difference can be seen with respect to the acoustic predictions in width, position and strength of the stop band. Furthermore, the measured insertion loss is rather flat for the higher frequency region (above $1200 \mathrm{~Hz}$ ), indicating a damped behaviour.

In view of a deeper insight into the mismatch between numerical predictions and acoustic measurements, the nominal enclosure was fabricated three additional times; the first enclosure $(1 \mathrm{~F}-1)$, shown in figure 25 was built as a demonstrator, next two similar enclosures $(1 \mathrm{~F}-2,1 \mathrm{~F}-3)$ were built 6 months later and a fourth enclosure $(1 \mathrm{~F}-4)$ was built again one month later together with the variations in design which will be discussed later. After fabrication of the fourth enclosure, all enclosures were measured after each other and under similar conditions. Figure 26 shows this measured insertion loss of the four enclosures. Although the four enclosures show a similar overall behaviour, clearly a shift in resonance frequencies and increased insertion loss zones can be seen. Enclosure (1F-1) and (1F-4) have a $5 \mathrm{~dB}$ difference in insertion loss increase as well as a $200 \mathrm{~Hz}$ shift in frequencies of the increased insertion loss zone. The measurements were repeated several times and the measurements showed to be consistent over time for the different enclosures.

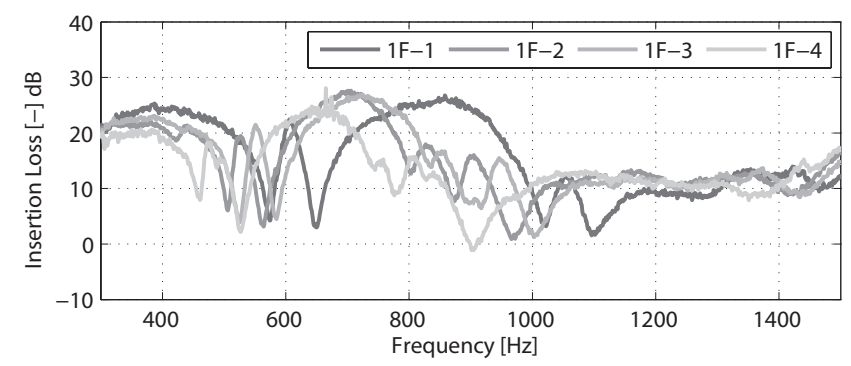

Figure 26: Comparison between measurements on 4 nominally equal enclosures built on different times. 
This difference between the enclosures is noticeable in the weight of the enclosures as well; the enclosures have a weight of 166, 150, 149 and $138 \mathrm{~g}$ respectively. Based on the models and the material parameters stated by the manufacturer, a weight of $176 \mathrm{~g}$ was expected. This clearly indicates a spread in material parameters for the different enclosures, which explain the different behaviours.

The remainder of this section discusses the measured and predicted behaviour of the variation of enclosures designed to investigate the effect of design parameters on the stop bands. Since the fourth nominal enclosure 1 F-4 was ordered together with these variations of design, it is expected that the fabrication scatter for this enclosure is less with respect to the other designs than for the other nominal enclosures fabricated; the fourth enclosure is thus chosen as benchmark case for comparisons. Although less spread in production between the enclosures is expected, it is worthwhile to point out that this enclosure differs most with the numerically predicted insertion loss and weight.

Given the large difference between enclosures resulting from the same production file, additional investigations into possible causes of these differences are added in appendix Appendix A. Based on the results of this appendix, the differences between the numerical results and experimental measurements are adressed and the possibility of modal updating is discussed in appendix Appendix B. From these investigation it is apparent that additive manufacturing is characterised by a large spread on material parameters, mainly on Young's modulus, both within one enclosures and between two enclosures. Through modal updating, it is shown that numerical models with global material parameters do not suffice to describe the detailed frequency behaviour of the enclosures, however, global displacement pattern are retrieved rather accurately.

\subsection{Variations of enclosures}

The large scatter, shown in figure 26, on the measurements of enclosures which are expected to be equal, indicates that care should be taken in comparing measurement results on different enclosures; it is difficult to discriminate 
between design effects and production effects. To reduce differences in production as much as possible, all enclosures were asked to be made on the same machine with three enclosures being built at the same time. Below is listed which enclosures were asked to be fabricated at the same time, between brackets the ratio of actual weight to expected weight for each enclosure is given:

- 1R (82\%), 1P (79\%) and 1S (79\%);

- $1 \mathrm{~F}(78 \%), 2 \mathrm{~F}(78 \%)$ and $2 \mathrm{M}(78 \%)$

- 1I (85\%), 1N (96\%) and 1C (81\%).

Although requested differently, it is known that at least the last three enclosures were fabricated separately.

To compare the different enclosure designs both numerically as well as experimentally, the measurement results for enclosure investigating a certain design parameter are grouped together and compared to the nominal enclosure. For each design parameter, the numerical predictions are first discussed and then compared to the measurement results.

\subsubsection{F-1R-1P: Number of resonant structures}

Enclosures $1 \mathrm{R}$ and $1 \mathrm{P}$ are designed to assess the importance of the number of resonators as well as the importance of periodicity. From the stop band analyses in section 5 it is expected that the stop bands for enclosures $1 \mathrm{R}$ and $1 \mathrm{P}$ are similar but smaller than the stop band of enclosure $1 \mathrm{~F}$.

The simulation results, shown in figure 27. confirm these expectations; the zone of increased insertion loss is smaller for enclosures $1 \mathrm{R}$ and $1 \mathrm{P}$ in agreement with the stop band prediction. Moreover from the simulation results it seems that the effect of periodicity is negligible; the predicted insertion loss for enclosure $1 \mathrm{R}$ and $1 \mathrm{P}$ are almost equal. Enclosure $1 \mathrm{~F}$ shows a stronger increase in insertion loss which is expected since this enclosure has a larger number of acting resonant structures.

Comparison of the measurement results shows the same trends: enclosures $1 \mathrm{R}$ and $1 \mathrm{P}$ show a similar behaviour. Differences in manufacturing accuracy 
could explain the small differences in insertion loss curves. Stop band zones can be indicated for enclosures $1 \mathrm{R}$ and $1 \mathrm{P}$, although these are not strongly pronounced.
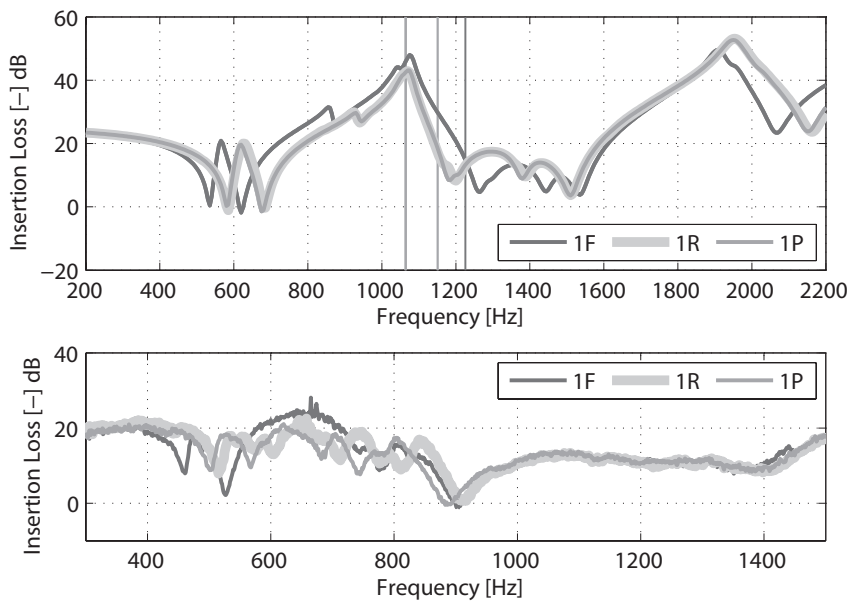

Figure 27: Top: Comparison between the numerically predicted insertion loss for enclosure $1 \mathrm{~F}$ and the enclosures with a reduced number of resonant structure (1R and $1 \mathrm{P})$. The narrow vertical lines indicate the predicted stop band zone. Bottom: The measured insertion loss.

\subsubsection{F-1C-1I: Skin location}

Enclosures 1C and 1I have a different skin location with respect to enclosure $1 \mathrm{~F}$; skin on the inside respectively on both sides of the core instead of on the outside. These changes in host structure allow for the analysis of two effects. Firstly both variations have less material which is exposed to the noise source and thus absorption effects can be investigated. This absorption effect is not included in the numerical models and can thus only be investigated by the measurement results. Secondly enclosure 1C is characterised by a different bending stiffness since in this case the core is not fully closed. This allows assessing whether or not this influences the stop band width. This effect was shown to be important in the unit cell based stop band prediction of table 4 , a narrower stop band is predicted for enclosure 1C.

Inspection of the simulation results, shown in figure 28, shows that overall the stop bands are very similar. Enclosure 1C shows a somewhat narrower stop 
band but this reduction is less pronounced as expected based on the unit cell analyses (section 5.3 .
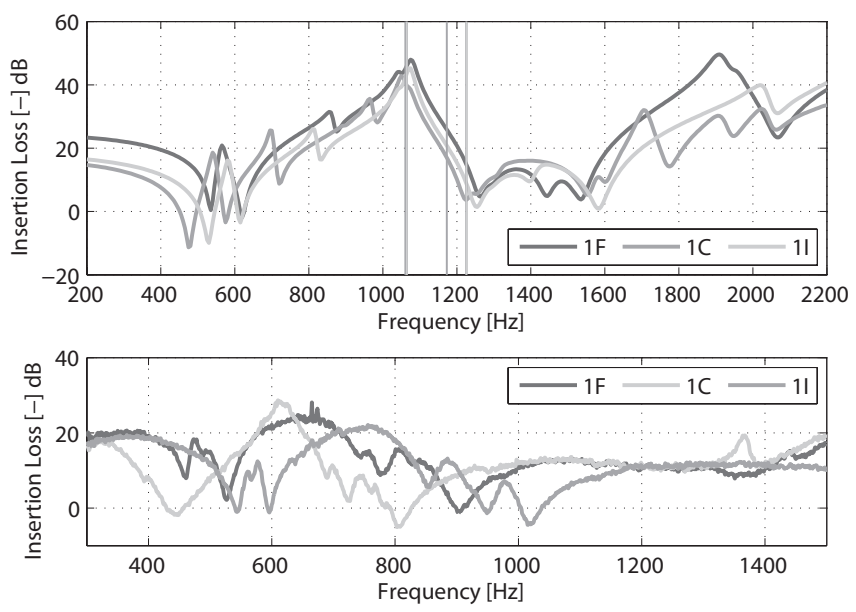

Figure 28: Top: Comparison between the numerically predicted insertion loss for enclosure $1 \mathrm{~F}$ and an enclosure with skin on both sides (1C) and skin on the inside (1I). The narrow vertical lines indicate the predicted stop band zone. Bottom: The measured insertion loss.

Inspection of the measurement results, figure 28, shows a stop band in all three enclosures as well. The stop bands, however, are found at different frequencies, given the difference in actual weight to expected weight for these enclosures, a strong difference between measurement results was expected. Since all three enclosures show stop band behaviour, it can be assumed that absorption of the material is not the driving factor in creating acoustic transmission reduction.

\subsubsection{F-1S: Different shape}

Enclosure 1S is built in view of investigating the importance of the shape of the enclosure. As can be seen from both the numerical as well as the measurement results in figure 29), the shape of the enclosure has an influence on the general acoustic behaviour but does not seem to be of importance for obtaining stop bands. It is interesting to note that enclosure 1S has only 260 resonant structures whereas enclosure $1 \mathrm{~F}$ has 320 resonant structures, both stop bands however are similar. This indicates that not the number of resonant structures but the relative weight of the resonant structures is of importance; the relative 
weight of the resonant structures to the host structure is the same for both enclosures.
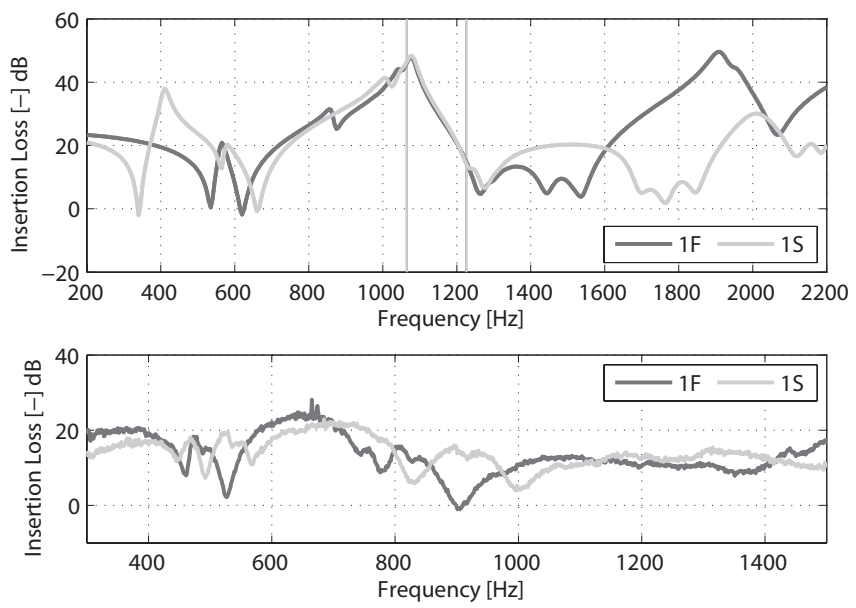

Figure 29: Top: Comparison between the numerically predicted insertion loss for enclosure $1 \mathrm{~F}$ and an enclosure with a different size (1S). The narrow vertical lines indicate the predicted stop band zone. Bottom: The measured insertion loss.

\subsubsection{F-2F-2M: Different resonant structures}

The control of stop band frequencies and combination of stop bands are investigated through enclosures $2 \mathrm{~F}$ and $2 \mathrm{M}$. The numerical simulations, shown in figure 30, follow the stop band prediction on unit cell level quite well; a different resonant structure leads to a stop band at a different frequency and combination of two resonant structures leads to two, narrower, stop bands.

Before the second stop band zone, a resonance dip around $1500 \mathrm{~Hz}$ seems to reduce the stop band width. This resonance peak is an acoustic resonance; around $1500 \mathrm{~Hz}$ the length of the enclosure equals half an acoustic wave. The strength of this resonance is also sensitive to the impedance layer representing the trim layer on the test set-up, indicating that this is indeed an acoustic resonance. Although this resonance dip seems to reduce the width of the stop band, the stop band actually reduces the strength of the resonance dip, which can be seen by comparison to the curve of the host structure in figure 25 which is $10 \mathrm{~dB}$ stronger in that frequency zone. 

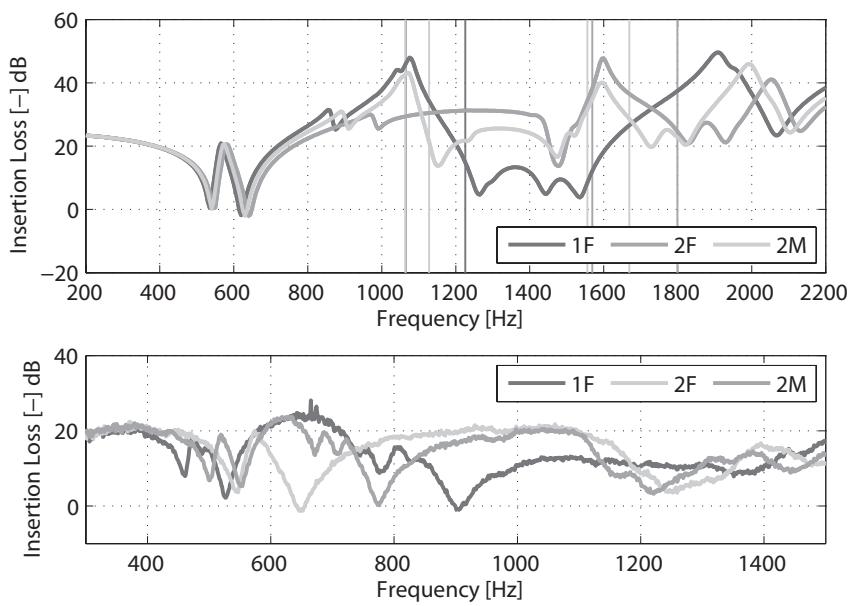

Figure 30: Top: Comparison between the numerically predicted insertion loss for enclosure $1 \mathrm{~F}$ and enclosures with a different resonant structure $(2 \mathrm{~F})$ or combination of both $(2 \mathrm{M})$. The narrow vertical lines indicate the predicted stop band zone. Bottom: The measured insertion loss.

Comparison of the measurements and the simulations confirms the predicted trends; enclosure $2 \mathrm{M}$ shows both zones of increased insertion loss of enclosure $1 \mathrm{~F}$ and $2 \mathrm{~F}$ but both zones are less pronounced. Since in measurements the stop band is found at lower frequencies, the acoustic resonance does not influence the second stop band zone.

\subsubsection{F-1N: No resonant structures}

To benchmark the effect of stop bands against a structure with an equivalent mass, enclosure $1 \mathrm{~N}$ was fabricated. This enclosure is a traditional enclosure built from flat panels. Due to the small dimensions of the enclosure, it should be noted that the acoustic response of the enclosure is still strongly stiffness driven. From both the numerical as experimental results, shown in figure 31 . the difference in acoustic response due to the lack of stop band behaviour in enclosure $1 \mathrm{~N}$ is apparent. To see and hear the difference between enclosure $1 \mathrm{~F}$ and $1 \mathrm{~N}$ in a movie, the interested reader is referred to a movie which can be seen by visiting the following link (http://youtu.be/t0ch_GsGaXg). 

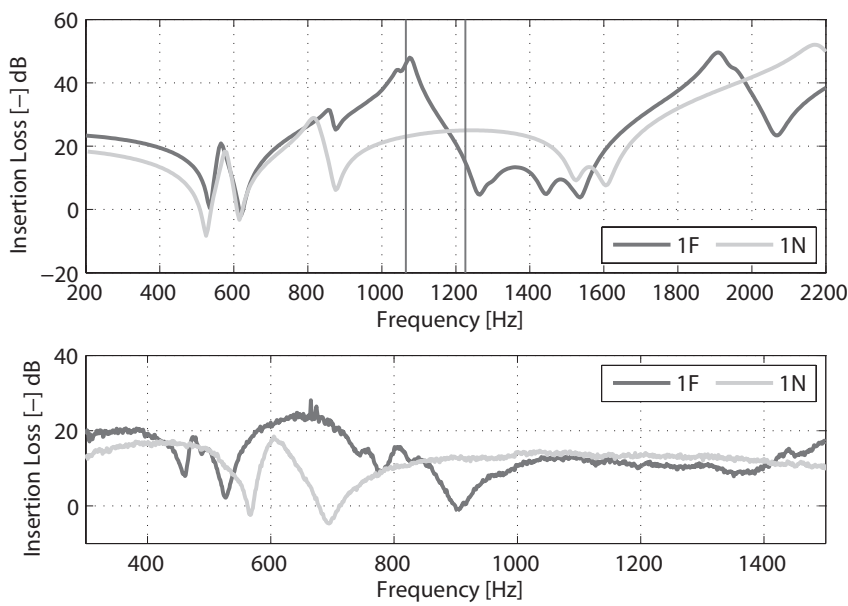

Figure 31: Top: Comparison between the numerically predicted insertion loss for enclosure $1 \mathrm{~F}$ and an enclosure built from flat panels $(1 \mathrm{~N})$. The narrow vertical lines indicate the predicted stop band zone. Bottom: The measured insertion loss.

\section{Conclusion}

This paper introduces a novel method to create resonant metamaterials for acoustic insulation; the inclusion of resonant structures within the core of a sandwich structure. Through the design of a set of demonstrators it is shown that this results in a frequency zone of increased acoustic insertion loss with respect to equivalent materials of the same weight besides the driving parameters are identified.

Stop band predictions based on unit cell modelling are compared to numerical simulations of the insertion loss of the demonstrators. Comparison between numerical predictions and measurements was hindered somewhat by the high degree of variation between the produced enclosures. Nevertheless, in general the numerically predicted trends correspond well with measurements. It can be concluded that:

- the strength and width of the stop band depend on the relative mass addition of the resonant structure with respect to the host structure,

- the resonance frequency of the resonant structure determines the location of the stop band, 
- the stop band effect does not depend on the size of the structure to which it is added,

- different resonant structures can be added to create multiple stop bands,

- the stop band does not depend on periodicity.

The material parameters identification of the different enclosure, however, indicates that care should be taken when using additive manufacturing vibroacoustical prototyping. The large scatter on material parameters, both within one enclosures as between different enclosures, indicates that SLS might not be the most suitable production technology to obtain resonance based metamaterials in a controlled fashion. Nevertheless, the methodology suffices to demonstrate the potential of stop bands and gives an insight in the design.

This metamaterial concept allows the combination of light weight, compact mass and good acoustic behaviour along with other technological benefits such as integration in structural parts, use in harsh environments and easy designable beneficial frequency ranges. To see and hear this potential in a movie, the interested reader is referred to a movie which can be seen by visiting the following link (http://youtu.be/tOch_GsGaXg).

\section{acknowledgements}

The authors would like to acknowledge the European Commission for their support through the ENLIGHT-project (http://www.project-enlight.eu/) and the KU Leuven Research Fund for their support through a IOF-Leverage project. Elke Deckers is a postdoctoral fellow of the Fund for Scientific Research Flanders (F.W.O.).

\section{bibliography}

[1] Allemang, R. J., 2003. The modal assurance criterion - twenty years of use and abuse. Sound and Vibration 37 (8), 14-23. 
[2] ASTM, 2003. D638 - 03: Standard test method for tensile properties of plastics.

[3] Brillouin, L., 1946. Wave propagation in periodic structures, 2nd Edition. McGraw-Hill Book Company.

[4] Claeys, C., Sas, P., Desmet, W., 2014. On the acoustic radiation efficiency of local resonance based stop band materials. Journal of Sound and Vibration 333 (14), 3203-3213.

[5] Claeys, C., Vergote, K., Sas, P., Desmet, W., 2013. On the potential of tuned resonators to obtain low-frequency vibrational stop bands in periodic panels. Journal of Sound and Vibration 332 (6), 1418-1436.

[6] Diaz, A., Haddow, A., Ma, L., 2005. Design of band-gap grid structures. Structural and Multidisciplinary Optimization 29 (6), 418-431.

[7] Ferry, J. D., 1980. Viscoelastic properties of polymers, 3rd Edition. Wiley.

[8] Goffaux, C., Sánchez-Dehesa, J., Yeyati, A., Lambin, P., Khelif, A., Vasseur, J., Djafari-Rouhani, B., 2002. Evidence of fano-like interference phenomena in locally resonant materials. Physical Review Letters 88 (22), 225502.

[9] Kittel, C., 2005. Introduction to Solid State Physics, 8th Edition. Wiley.

[10] Langley, R., 1993. A note on the force boundary conditions for twodimensional periodic structures with corner freedoms. Journal of Sound and Vibration 167, 377-381.

[11] Lemoult, F., Kaina, N., Fink, M., Lerosey, G., 2012. Wave propagation control at the deep subwavelength scale in metamaterials. Nature Physics $9,55-60$.

[12] Liu, L., Hussein, M., 2012. Wave motion in periodic flexural beams and characterization of the transition between Bragg scattering and local resonance. Journal of Applied Mechanics 79 (1), 011003. 
[13] Pendry, J. B., Holden, A. J., Robbins, D., Stewart, W., 1999. Magnetism from conductors and enhanced nonlinear phenomena. Microwave Theory and Techniques, IEEE Transactions on 47 (11), 2075-2084.

[14] Sigmund, O., Jensen, J. S., 2003. Systematic design of phononic band-gap materials and structures by topology optimization. Philosophical Transactions of The Royal Society 361, 1001-1019.

[15] Vivolo, M., 2013. Vibro-acoustic characterization of lightweight panels by using a small cabin. Ph.D. thesis, KU Leuven, Departement Werktuigkunde.

[16] Wadley, H. N., 2006. Multifunctional periodic cellular metals. Philosophical Transactions of the Royal Society A: Mathematical, Physical and Engineering Sciences 364 (1838), 31-68.

[17] Wang, G., Wen, X., Wen, J., Shao, L., Liu, Y., 2004. Two-dimensional locally resonant phononic crystals with binary structures. Physical Review Letters 93 (15), 154302.

[18] Xiao, Y., Wen, J., Wen, X., 2012. Flexural wave band gaps in locally resonant thin plates with periodically attached spring-mass resonators. Journal of Physics D: Applied Physics 45 (19), 195401.

\section{Appendix A. Determination of material parameters}

Since every production process is characterised by variation in parameters, some mechanisms are included in the enclosure design to retrieve the material parameters in a non-destructive way. To this end extra test specimens are produced together with the enclosures. Test specimens for tensile testing (Figure A.32 right) allow the determination of the material properties of the produced parts.

Connecting the test specimens to the outer edges of the enclosures (Figure A.32 left) ensures that the test specimens are printed at the same location 
as the enclosure and in the correct direction with respect to the enclosures. The test specimens are added according to the three principal axes of the enclosure and dedicated nomenclature of the test specimens allows linking each specimen with the associated enclosure and direction afterwards. Small material strips are used as connection between enclosure and test samples since these can easily be removed in a after production.

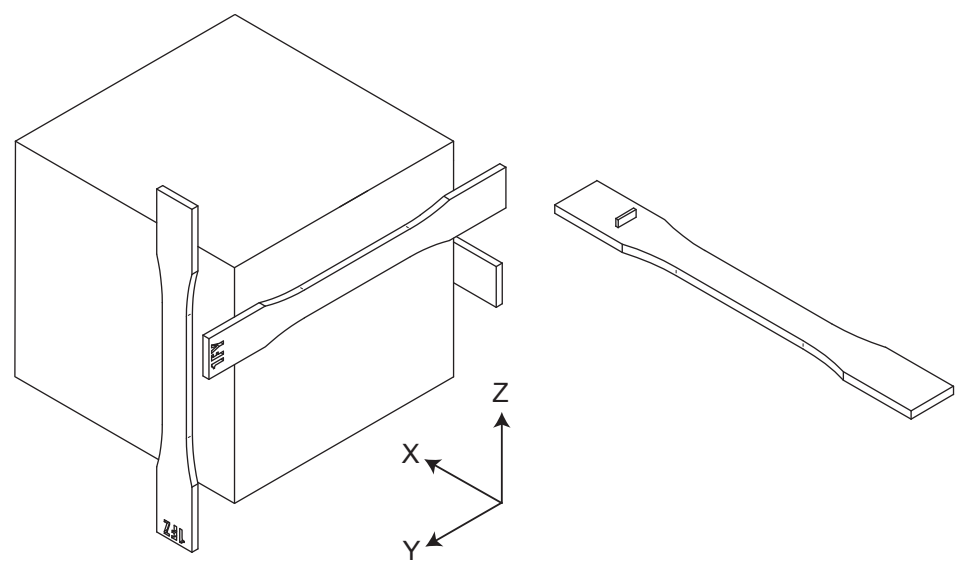

Figure A.32: CAD drawings of the production file of an enclosure with connected test specimens (left). Test specimens for tensile testing (right) are connected according to the three principal axes of the enclosure.

It was indicated that the SLS inherently leads to anisotropic material parameters and that the material process can be highly influenced by parameters such as workload on the machine, powder quality and laser scanning patterns. This stands in strong contrast with the goal of achieving a metamaterial with identical resonant structures.

It should be noted that, due to the layer wise building method of SLS, a high degree of anisotropy is expected. Processing the production file in the original positioning of the CAD file (Fig. A.33 left) would lead to differences in resonance frequency of the resonant structures in different panels of the enclosures: the resonant structures in the side panel are built perpendicular to the layer direction while the resonant structures in the top panel are positioned parallel to the layer direction. If the changes between the resonant structures in 
top and side plane become too large, the different panels could show different stop band behaviour. By changing the orientation of the enclosure by rotating the enclosure an angle of $45^{\circ}$ around both $x$ - and $y$-axis (Fig. A.33 right), all resonant structures are built in a more similar orientation with respect to the building platform and thus the layer direction, minimizing the chance of scatter in resonance frequencies in the resonant structures of one enclosure.
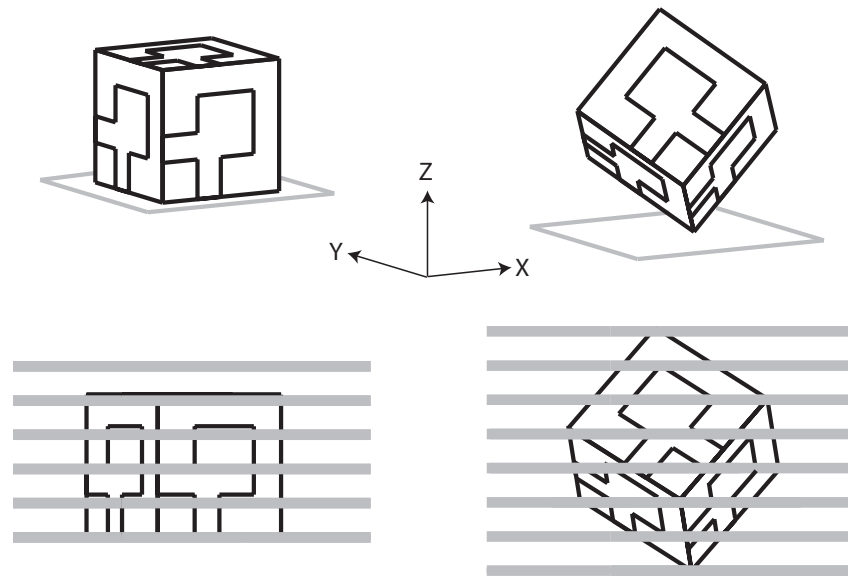

Figure A.33: Sketch indicating the resonant structure direction in an enclosure $(-)$ with respect to the build platform of an SLS machine (-). Top: On the left the original CAD positioning, on the right the same part but rotated an angle of $45^{\circ}$ around both $x$ - and $y$-axis. Bottom: a side view of the top figure, indicating the layer direction (thick grey lines) during production.

To determine the material parameter scatter within and between different enclosures, the designed material test samples are analysed. A tensile test allows the determination of Young's modulus and Poisson's ratio. Afterwards a small material part of the test sample is used to determine the density of the material. This section first describes the test samples, followed by a description of the measurement devices and a discussion of the measurement results.

\section{Appendix A.1. Test samples}

The test specimens for tensile testing are designed according to the ASTM D638 standard [2]. Figure A.34 gives the layout of the test sample; a small material strip is added to connect the test sample with the enclosure during 
production. The connection strip is removed prior to tensile testing.

The samples have a nominal thickness of $3 \mathrm{~mm}$ and a width of the narrow section of $13 \mathrm{~mm}$. The exact dimensions of the samples are necessary to determine the correct stresses and elongations during tensile testing. The thickness and width of each sample was measured with a Vernier calliper and the measured geometry was used in post-processing of the data.

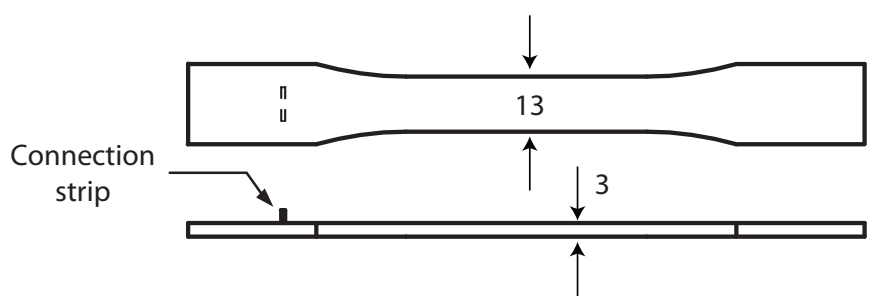

Figure A.34: Test sample for material testing through tensile testing. Dimensions are shown in $\mathrm{mm}$. Top: top view, bottom: side view.

To perform the density tests small material samples are required; parts of the samples for tensile testing are used to this end. The selected parts are outside the clamping part of the test sample since these parts can be both dirty and compressed due to the mounts of the tensile test device. Furthermore the same part of the test samples is selected for all samples, such that the location of the density measurement is the same for all enclosures.

\section{Appendix A.2. Measurement devices}

An Instron 4505 frame with an Instron 2518 load cell is used to perform the tensile testing; a force cell registers the force during the test while two extensometers are used to measure the change in length and width of the test samples (Fig. A.35). The measurements are performed as described in the ASTM D638 standard 2]. These measurements allow determining Young's modulus and Poisson's ratio.

According to the standard the Young's modulus should be calculated by locating the linear part of the load-extension curve and dividing the difference in stress corresponding to any part of this section by the corresponding difference in 


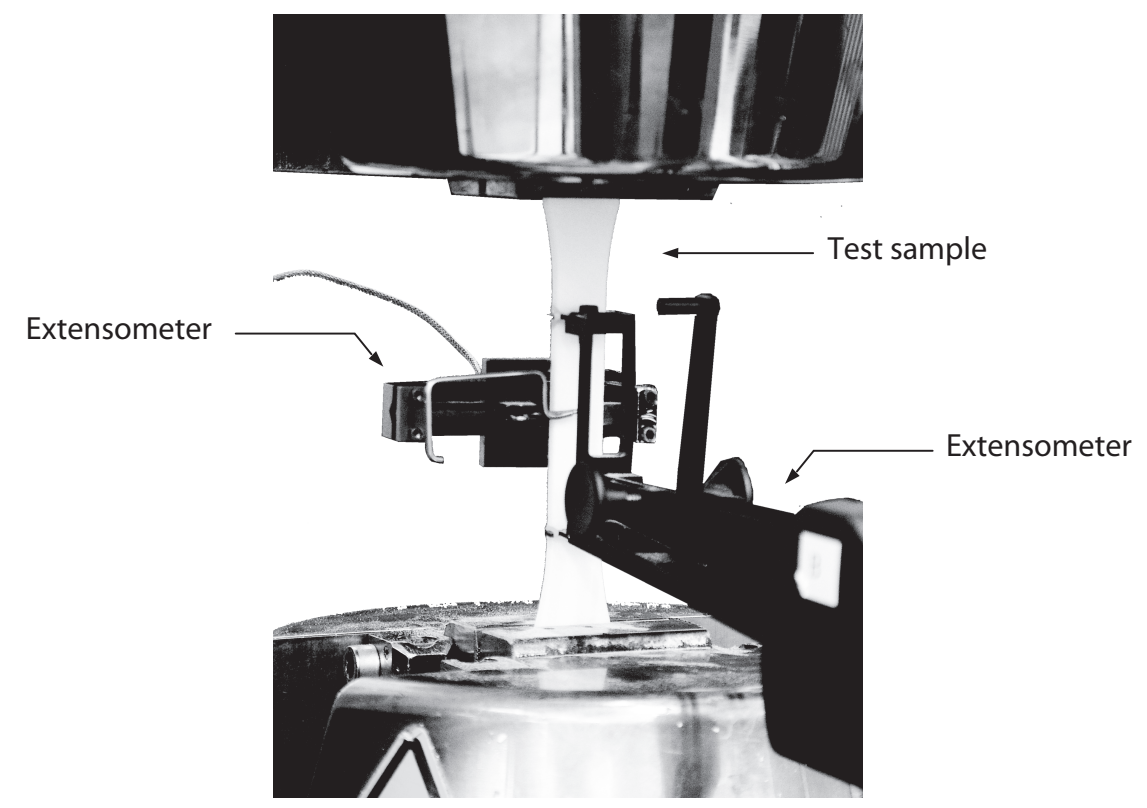

Figure A.35: Picture of a tensile test and the set-up.

strain of this section. In the post-processing software the linear part is identified as a region where the change in gradient of a consecutive number of measurement points is within a predefined interval. Next a straight line is fitted through the entire linear region and the slope of this line is taken as measured Young's modulus.

To obtain the Poison's ratio the slope of the longitudinal and lateral strain with respect to the force in the linear region of the stress-strain curve is calculated based on a least square fit of a line. The standard describes that the Poison's ratio equals the ratio of this lateral strain slope with respect to this longitudinal strain slope.

To determine the density an Acculab Atilon laboratory scale with a Sartorius YDK01 LP density determination kit is used. This set-up allows the determination of the density of a material part based on the buoyancy principle. The weight of a material part in air is compared with the weight of that material part when immersed in ethanol. Based on the difference in weight, the density of the solid can be determined. Care should be taken that the samples are clean 
and that no air bubbles are stuck to the outside of the surface.

\section{Appendix A.3. Measurement results}

Due to the destructive nature of tensile testing, a repetition check for the measurements could not be performed for the tensile tests. A repetition check on one of the test samples for density testing indicated the repeatability for 5 measurements during the time span of the test on all test samples: an estimated standard deviation of $1.06\left[\frac{\mathrm{kg}}{\mathrm{m}^{3}}\right]$ was found around a mean of $965.86\left[\frac{\mathrm{kg}}{\mathrm{m}^{3}}\right]$.

Figure A.36 gives an overview of the material parameters retrieved during material testing. The averaged value for each enclosure is given together with the measurements for each principal direction. The averaged results for each enclosure are given in table A.5. It should be noted that:

- The test sample for the $z$-direction of enclosure $1 P$ has not been tested due to a production error in the test sample.

- During the measurements of the Poisson's ratio for the $z$-direction of enclosure $1 F$ and the $y$-direction of enclosure $2 F$ the extensometer came loose, making this data unsuitable for post-processing.

\begin{tabular}{c|ccccccccc}
\hline & $1 \mathrm{~F}$ & $1 \mathrm{R}$ & $1 \mathrm{P}$ & $1 \mathrm{~S}$ & $1 \mathrm{C}$ & $1 \mathrm{I}$ & $2 \mathrm{M}$ & $2 \mathrm{~F}$ & $1 \mathrm{~N}$ \\
\hline $\mathrm{E}[\mathrm{MPa}]$ & 1092 & 1292 & 1413 & 1202 & 1216 & 1374 & 1187 & 996 & 1422 \\
$\nu[-]$ & 0.36 & 0.40 & 0.41 & 0.36 & 0.34 & 0.39 & 0.41 & 0.36 & 0.41 \\
$\rho\left[\frac{\mathrm{kg}}{\mathrm{m}^{3}}\right]$ & 956 & 968 & 968 & 965 & 962 & 975 & 957 & 963 & 972 \\
\hline
\end{tabular}

Table A.5: Overview of the average measured Young's modulus $(E)$, Poisson's ratio $(\nu)$ and density $(\rho)$ for each enclosure. The averages are obtained as the result of measurements of a test sample according to each principal direction of an enclosure.

Trends in and sources of scatter on parameters of the SLS production process is a research field in itself. The data in this section is determined in order to assess the material parameters in view of getting a feel for the spread in production process. Some interesting things to note, which are not investigated further in this paper are: 

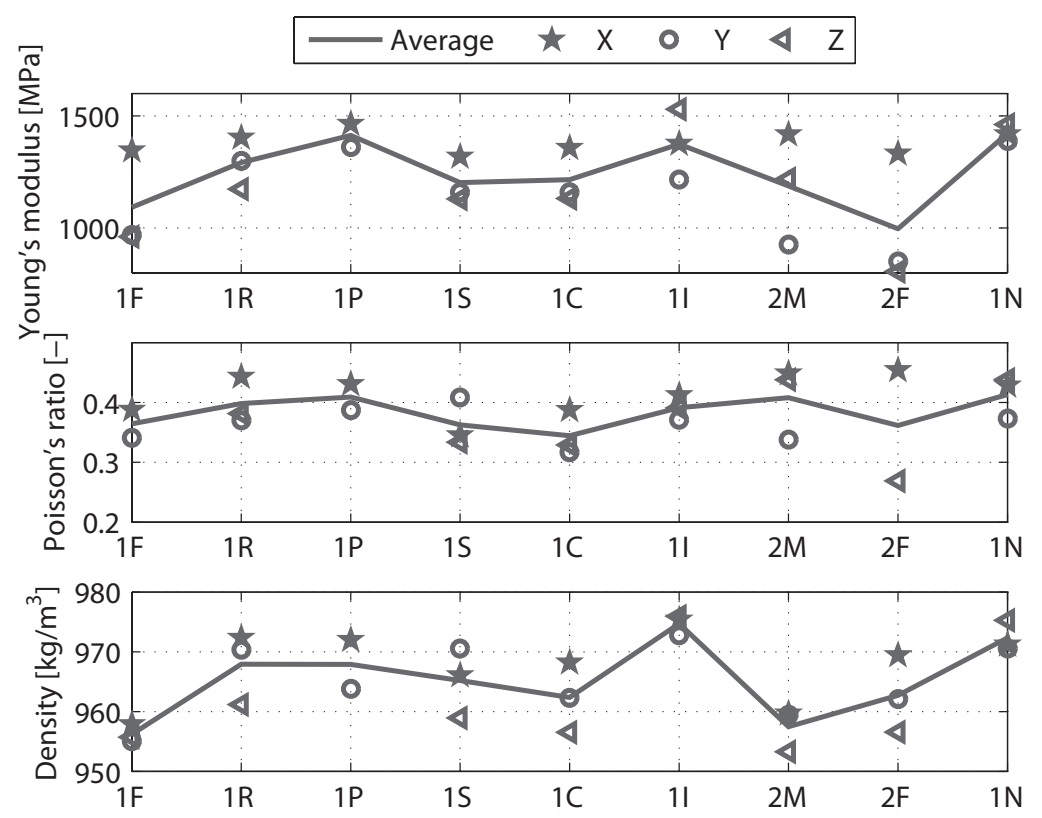

Figure A.36: The measured Young's modulus, Poisson's ratio and density for each enclosure. The measurements on a test sample according to each principal direction of an enclosure are given together with the average of the measurements of the three principal directions for each enclosure.

- The measurement results for the Young's modulus show that the variation in $x$-direction between different enclosures is in general less than the variation between the different principal axes of the enclosures. Figure A.37 gives an overview of the stress-strain curves for the different test samples and clearly illustrates this point.

- The average measured Young's moduli shows a similar trend as the average measured density.

- The measured Young's moduli are well below the minimal value of $1500 \mathrm{MPa}$ as prescribed by the manufacturer, who set forwards a Young's modulus of $1650 \pm 150 \mathrm{MPa}$

- Enclosure $1 N$ shows the least scatter on material parameters. It is worth- 
while to point out that this is also the only enclosure without resonant structures; this might, however, be a coincidence.

- The obtained density through testing of the materials samples does not correspond with the actual weight of the enclosures. For enclosure $1 \mathrm{~N}$ the prediction based on the measured density is acceptable; $163 \mathrm{~g}$ instead the actual measured mass of $169 \mathrm{~g}$. For all other enclosure this difference is a lot larger, for instance $173 \mathrm{~g}$ for the predicted weight against $138 \mathrm{~g}$ in reality for the nominal enclosure. This might indicate a lower sinter quality and thus a higher porosity in the enclosures then in the test samples. A higher porosity could in turn lead to a higher scatter on material parameters.
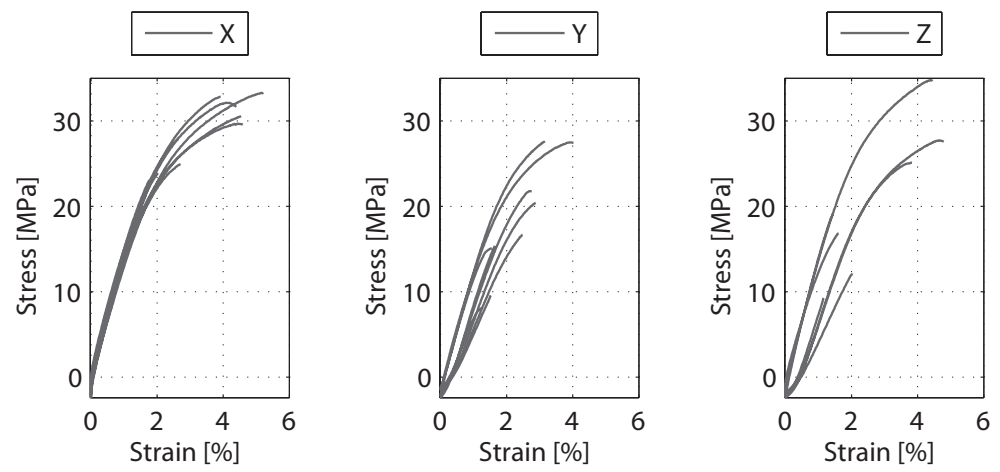

Figure A.37: Overview of the stress-strain curves of the different test samples.

\section{Appendix A.4. conclusion}

This section illustrates the high variation in and material parameters both within one produced part as between parts. This forms a major hurdle for stop band design. In view of using resonant metamaterials as acoustic insulation for targeted frequency ranges, it is crucial to come up with a reliable production process in order to obtain consistent and predictable stop band frequencies. It is interesting to note as well, that the part with a simple geometry, enclosure $1 N$, shows less variation then the more complex enclosures. 


\section{Appendix B. Modal updating}

\section{Appendix B.1. Modal identification}

In the context of numerical updating, modal identification is often applied; during modal identification the structural modes of a test structure are measured and compared with the structural modes predicted by a numerical model. Using this form of updating has as advantage that complexity of the numerical model is reduced since the acoustical part of the model can be omitted. Therefore it is chosen to start with updating of a purely structural model.

Enclosure $1 N$ is chosen to perform the updating campaign. Next to being easier to model, this enclosure shows the least spread on material parameters. Given the modelling assumptions of isotropic material with constant geometric and material parameters throughout the entire enclosure, it can be assumed that a low scatter on material parameters increases the chances of a successful match between model and test.

To obtain the structural modes in a test, enclosure $1 N$ is freely suspended with a bungee (Fig. B.38). A roving hammer excitation was used and two lightweight accelerometers (type PCB 352A24 with a weight of 0.8 gram a piece) were attached as reference to obtain the mode shapes of the enclosure. As input points for hammer excitation 10 points on each side-panel and 9 points on the top of the enclosure are chosen (black dots on Fig. B.38).

The numerical modes are obtained through the structural model of section 6 . updated according to the data from table A.5. The mass of the accelerometers is taken into account as localised discrete masses in the model of the enclosure.

The measured modes are compared with the modes obtained from the numerical model by means of a MAC-matrix 1] (table B.6). The MAC-matrix indicates that the mode shapes (Fig. B.39) are well captured by the model, the frequencies of the mode shapes however seem to be underestimated. Besides the MAC-matrix, table B.6 indicates for each mode the relative difference in measured and simulated resonance frequency with respect to the measured res-

onance frequency $\left(\frac{\Delta f}{f_{\text {exp }}}\right)$ and the amount of modal damping (Damp.) measured. The damping is given as percentage of critical modal damping. 

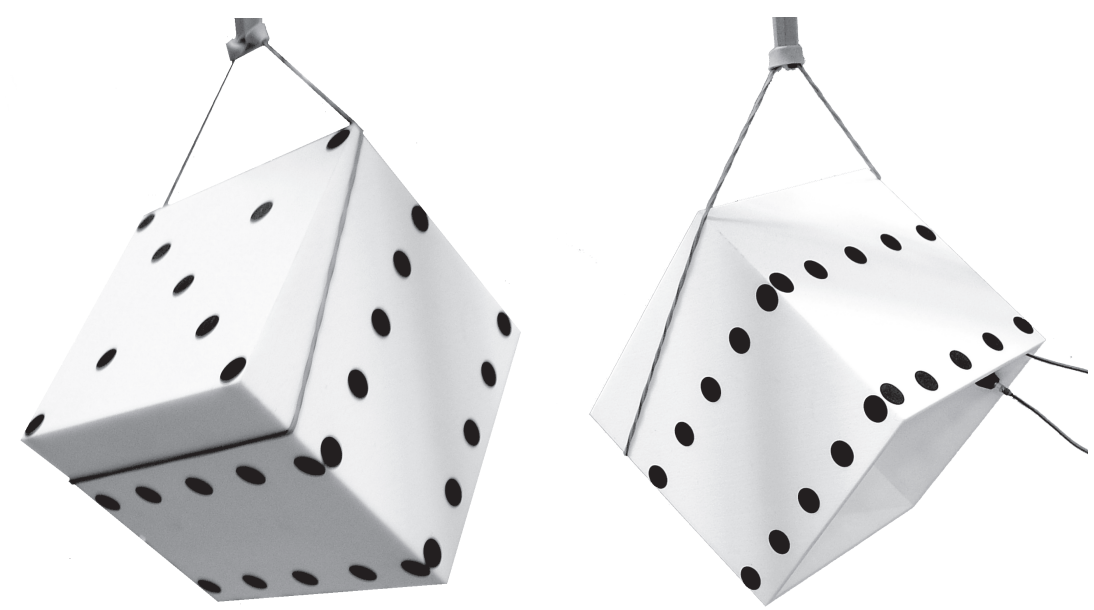

Figure B.38: Pictures of the suspension of enclosure $1 N$ in view of modal testing. The black dots indicate the impact points for hammer excitation, on the outside the bungees for suspension are visible and on the inside of the enclosure on the right picture the cables of the accelerometers can be seen.

\section{Appendix B.2. Discussion}

Appendix B.2.1. Frequency mismatch

The mismatch between resonance frequencies predicted by the numerical model with respect to the measured resonance frequencies could be compensated by tuning the material or geometrical parameters. However, since the applied parameters are measured, this form of model updating implies model compensation rather than model updating. Furthermore, table B.6 shows that modes 7 to 10 suffer a relative higher mismatch $\left(\frac{\Delta f}{f_{\text {exp }}}\right)$ than the other modes. Modes 7 to 10 are higher-order modes of modes 2 to 5 (Fig. B.39); indicating that the difference in frequencies is rather due to local than global effects.

The difference in resonance frequencies was further investigated both numerically as well as experimentally. A different set-up was built to investigate the effect of the suspension of the enclosure and a numerical model using solid elements was compared to the shell-element models. Both investigations could not resolve the increase in relative error in frequency between numerical predictions and experiments. From this it seems that a model with globally defined geometrical and material parameters falls short in describing the exact frequency 


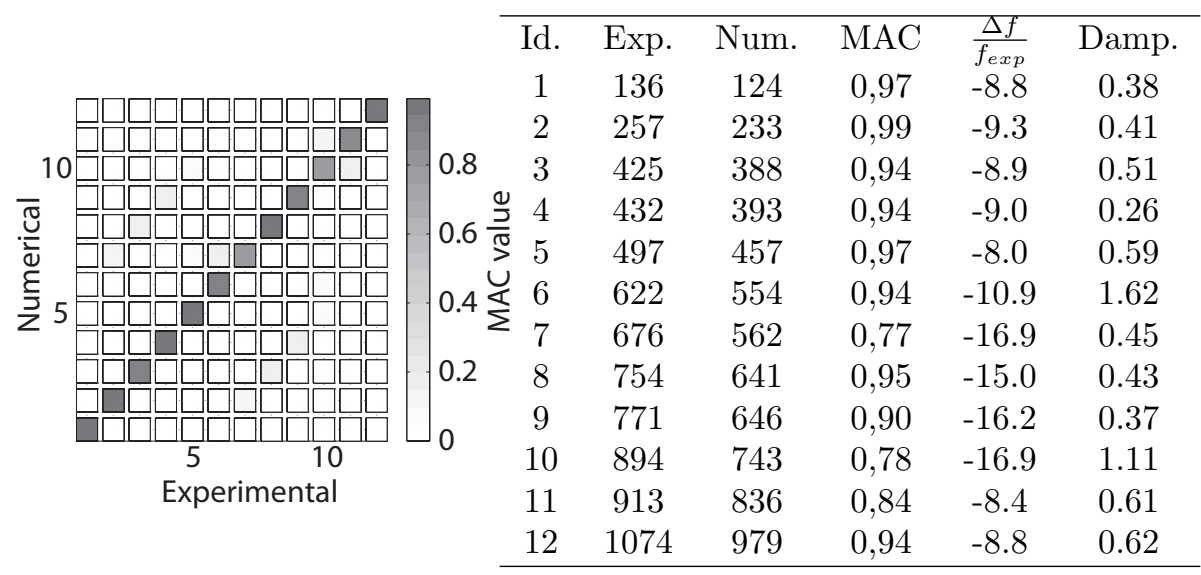

Table B.6: Comparison between experimental and numerical modes for enclosure $1 N$ in freely suspended conditions. All frequencies are given in $[\mathrm{Hz}]$, the damping is expressed as percentage of critical modal damping.
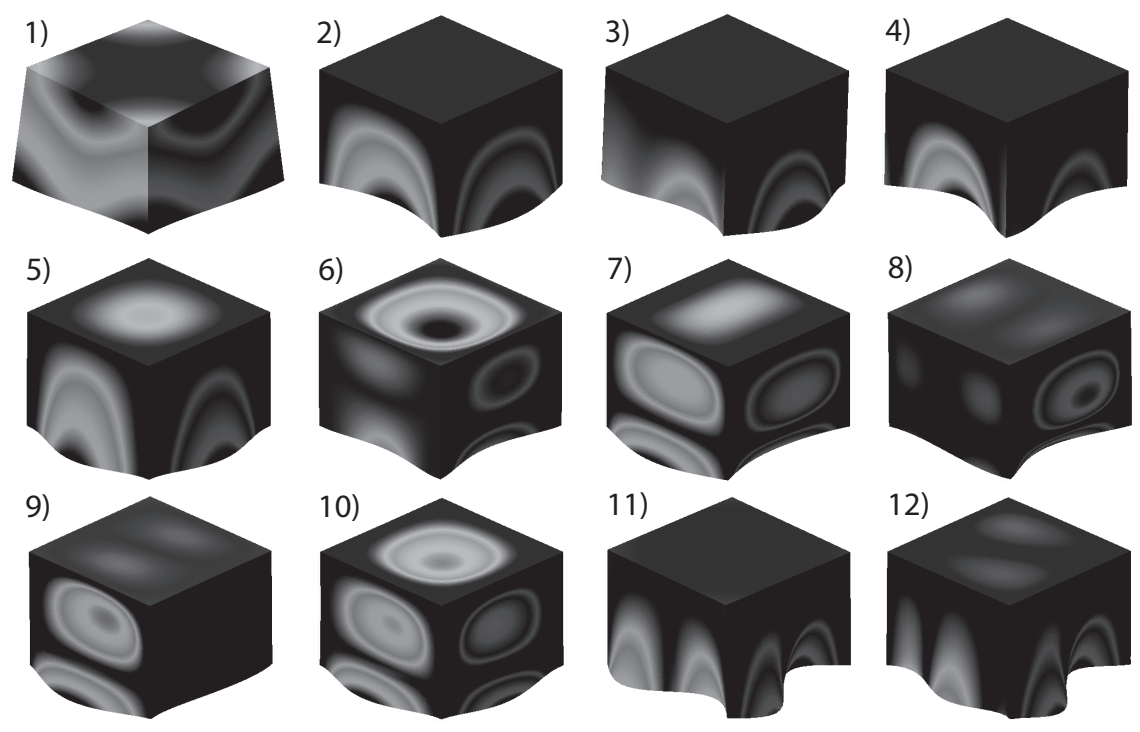

Figure B.39: Visualisation of the first 12 simulated mode shapes of enclosure $1 \mathrm{~N}$. The deformed mode shapes are shown and coloured according to the amplitude of the displacements $([\mathrm{m}])$. 
behaviour of the enclosures. However, given the good correlation between the mode shapes, the vibrational behaviour of the enclosure seems to be well captured.

\section{Appendix B.2.2. Damping}

The acoustic measurements on enclosure $1 \mathrm{~N}$ up to a frequency of $1000 \mathrm{~Hz}$ (Fig. 31) show clear resonance peaks, indicating low damping values. This corresponds well with the rather low damping values obtained from the modal identification. The overall behaviour in terms of sharpness of the peaks in the considered frequency regime corresponds quite well with the numerical predictions for which $1 \%$ of modal damping was assumed for all modes. In view of getting an estimate of the acoustic behaviour of the enclosure, the amount of structural damping used in the model seems justified.

In case of resonant inclusions, the comparison between acoustic measurements and simulations in terms of damping corresponds well outside the stop band region and below $1000 \mathrm{~Hz}$ (Fig. 25).

In order to obtain a better model of the damping (and the Young's modulus), Dynamic Mechanical Analysis (DMA) can be applied [7. This technique is often used for characterisation of viscoelastic materials and allows obtaining the complex modulus (stiffness and damping) with respect to a changing frequency or temperature. The material characteristics are derived by relating the measured strain in a material due to the application of a sinusoidal stress. Obtaining an exact material model of the materials used during additive manufacturing, however, is outside of the scope of this paper.

Appendix B.3. conclusion

Through an example of model updating of enclosure $1 \mathrm{~N}$, the enclosure which is easiest to model and has fairly constant material parameters, it is shown that

numerical models with global material parameters and do not suffice to describe the detailed frequency behaviour of the enclosures. 